\title{
Solving the At-Most-Once Problem with Nearly Optimal Effectiveness
}

\author{
Sotirios Kentros ${ }^{\mathrm{a}, 1}$, Aggelos Kiayias ${ }^{\mathrm{a}, 2}$ \\ ${ }^{a}$ Computer Science and Engineering, University of Connecticut, Storrs, USA
}

\begin{abstract}
We present and analyze a wait-free deterministic algorithm for solving the at-most-once problem: how $m$ shared-memory fail-prone processes perform asynchronously $n$ jobs at most once. Our algorithmic strategy provides for the first time nearly optimal effectiveness, which is a measure that expresses the total number of jobs completed in the worst case. The effectiveness of our algorithm equals $n-2 m+2$. This is up to an additive factor of $m$ close to the known effectiveness upper bound $n-m+1$ over all possible algorithms and improves on the previously best known deterministic solutions that have effectiveness only $n-\log m \cdot \mathrm{o}(n)$. We also present an iterative version of our algorithm that for any $m=\mathrm{O}(\sqrt[3+\epsilon]{n / \log n})$ is both effectiveness-optimal and work-optimal, for any constant $\epsilon>0$. We then employ this algorithm to provide a new algorithmic solution for the Write-All problem which is work optimal for any $m=\mathrm{O}(\sqrt[3+\epsilon]{n / \log n})$.
\end{abstract}

Keywords: at-most-once problem, task allocation, write-all, I/O automata, asynchronous shared memory, deterministic algorithms, distributed computing

\section{Introduction}

The at-most-once problem for asynchronous shared memory systems was introduced by Kentros et al. [26] as the problem of performing a set of $n$ jobs by $m$ fail-prone processes while maintaining at-most-once semantics.

The at-most-once semantic for object invocation ensures that an operation accessing and altering the state of an object is performed no more than once. This semantic is among the standard semantics for remote procedure calls (RPC) and method invocations and it provides important means for reasoning about the safety of critical applications. Uniprocessor systems may trivially provide solutions for at-most-once semantics by implementing a central schedule for operations.

\footnotetext{
Email addresses: skentros@engr.uconn. edu (Sotirios Kentros), aggelos @kiayias. com (Aggelos Kiayias)

${ }^{1}$ Research supported in part by the State Scholarships Foundation of Greece.

${ }^{2}$ Research supported in part by NSF awards 0831304, 0831306 and EU projects RECUP and CODAMODA

${ }^{3}$ NOTICE: This is the authors version of a work that was accepted for publication in Theoretical Computer Science. Changes resulting from the publishing process, such as peer review, editing, corrections, structural formatting, and other quality control mechanisms may not be reflected in this document. Changes may have been made to this work since it was submitted for publication. A definitive version was subsequently published in Theoretical Computer Science, [Volume 496, 22 July 2013] DOI:10.1016/j.tcs.2013.04.017
} 
The problem becomes very challenging for autonomous processes in a system with concurrent invocations on multiple objects. At-most-once semantics have been thoroughly studied in the context of at-most-once message delivery [8, 30, 33] and at-most-once process invocation for RPC [6, 31, 37]. However, finding effective solutions for asynchronous shared-memory multiprocessors, in terms of how many at-most-once invocations can be performed by the cooperating processes, is largely an open problem. Solutions for the at-most-once problem, using only atomic read/write memory, and without specialized hardware support such as conditional writing, provide a useful tool in reasoning about the safety properties of applications developed for a variety of multiprocessor systems, including those not supporting bus-interlocking instructions and multi-core systems. Specifically, in recent years, attention has shifted from increasing clock speed towards chip multiprocessing, in order to increase the performance of systems. Because of the differences in each multi-core system, asynchronous shared memory is becoming an important abstraction for arguing about the safety properties of parallel applications in such systems. In the next years, one can expect chip multiprocessing to appear in a wide range of applications, many of which will have components that need to satisfy at-most-once semantics in order to guarantee safety. Such applications may include autonomous robotic devices, robotic devices for assisted living, automation in production lines or medical facilities. In such applications performing specific jobs at-most-once may be of paramount importance for safety of patients, the workers in a facility, or the devices themselves. Such jobs could be the triggering of a motor in a robotic arm, the activation of the X-ray gun in an X-ray machine, or supplying a dosage of medicine to a patient.

Perhaps the most important question in this area is devising algorithms for the at-most-once problem with good effectiveness. The complexity measure of effectiveness [26] describes the number of jobs completed (at-most-once) by an implementation, as a function of the overall number of jobs $n$, the number of processes $m$, and the number of crashes $f$. The only deterministic solutions known, exhibit very low effectiveness $\left(n^{\frac{1}{\log m}}-1\right)^{\log m}$ (see [26]) which for most choices of the parameters is very far from optimal (unless $m=\mathrm{O}(1)$ ). Contrary to this, the present work presents the first wait-free deterministic algorithm for the at-most-once problem which is optimal up to additive factors of $m$. Specifically our effectiveness is $n-(2 m-2)$ which comes close to an additive factor of $m$ to the known upper bound over all possible algorithms for effectiveness $n-m+1$ (from [26]). We also demonstrate how to construct an algorithm which has effectiveness $n-\mathrm{O}\left(m^{2} \log n \log m\right)$ and work complexity $\mathrm{O}\left(n+m^{3+\epsilon} \log n\right)$, and is both effectiveness and work optimal when $m=\mathrm{O}(\sqrt[3+\epsilon]{n / \log n})$, for any constant $\epsilon>0$ (work complexity counts the total number of basic operations performed by the processes). Finally we show how to use this algorithm in order to solve the Write-All problem [23] with work complexity $\mathrm{O}\left(n+m^{3+\epsilon} \log n\right)$.

Related Work: A wide range of works study at-most-once semantics in a variety of settings. Atmost-once message delivery [8, 30, 33, 38] and at-most-once semantics for RPC [6, 31-33, 37], are two areas that have attracted a lot of attention. Both in at-most-once message delivery and RPCs, we have two entities (sender/client and receiver/server) that communicate by message passing. Any entity may fail and recover and messages may be delayed or lost. In the first case one wants to guarantee that duplicate messages will not be accepted by the receiver, while in the case of RPCs, one wants to guarantee that the procedure called in the remote server will be invoked at-most-once [37].

In Kentros et al. [26], the at-most-once problem for asynchronous shared memory systems and the correctness properties to be satisfied by any solution were defined. The first algorithms 
that solve the at-most-once problem were provided and analyzed. Specifically they presented two algorithms that solve the at-most-once problem for two processes with optimal effectiveness and a multi-process algorithm, that employs a two-process algorithm as a building block, and solves the at-most-once problem with effectiveness $n-\log m \cdot o(n)$ and work complexity $\mathrm{O}(n+m \log m)$. Subsequently Censor-Hillel [22] provided a probabilistic algorithm in the same setting with optimal effectiveness and expected work complexity $\mathrm{O}\left(n m^{2} \log m\right)$ by employing a probabilistic multi-valued consensus protocol as a building block.

Following the conference version of this paper [25] and motivated by the difficulty of implementing wait-free deterministic solutions for the at-most-once problem that are effectiveness optimal, Kentros et al. [24] introduced the strong at-most-once problem and studied its feasibility. The strong at-most-once problem refers to the setting where effectiveness is measured only in terms of the jobs that need to be executed and the processes that took part in the computation and crashed. The strong at-most-once problem demands solutions that are adaptive, in the sense that the effectiveness depends only on the behavior of processes that participate in the execution. In this manner trivial solutions are excluded and, as demonstrated in [24], processes have to solve an agreement primitive in order to make progress and provide a solution for the problem. Kentros et al. [24] prove that the strong at-most-once problem has consensus number 2 as defined by Herlihy [21] and observe that it belongs in the Common 2 class as defined by Afek et al. [1]. As a result, there exists no wait-free deterministic solution for the strong at-most-once problem in the asynchronous shared memory model, using atomic read/write registers. Kentros et al. [24] present a randomized $k$-adaptive effectiveness optimal solution for the strong at-most-once problem, with expected work complexity of $\mathrm{O}\left(n+k^{2+\epsilon} \log n\right)$ for any small constant $\epsilon$, where $k$ the number of processes that participate in the execution.

Di Crescenzo and Kiayias in [11] (and later Fitzi et al. [14]) demonstrate the use of the at-most-once semantic in message passing systems for the purpose of secure communication. Driven by the fundamental security requirements of one-time pad encryption, the authors partition a common random pad among multiple communicating parties. Perfect security can be achieved only if every piece of the pad is used at most once. The authors show how the parties maintain security while maximizing efficiency by applying at-most-once semantics on pad expenditure.

Ducker et al. [12] consider a distributed task allocation problem, where players that communicate using a shared blackboard or an arbitrary directed communication graph, want to assign the tasks so that each task is performed exactly once. They consider synchronous execution without failures and examine the communication and round complexity required to solve the problem, providing relevant lower and upper bounds. If crashes are introduced in their model, the impossibility results from Kentros et al. [26] will apply to the at-most-once version of their problem.

Another related problem is the semi-matching problem [7, 10, 20]. The semi-matching problem known also as the load balancing problem has been extensively studied under various names in the network scheduling literature. Recently it has received renewed attention after a paper by Harvey et al. [20], where the name semi-matching was introduced. Semi-matching can be seen as an abstraction of the problem of matching clients with servers, each of which can process a subset of clients. The goal is to match each client with at-most-one server. Clients and servers are abstracted as the vertices of a bipartite graph, and a synchronous, failure-free, message-passing model of computation is assumed, where edges represent communication links.

One can also relate the at-most-once problem to the consensus problem [13, 21, 29, 35]. Indeed, consensus can be viewed as an at-most-once distributed decision. Another related problem 
is process renaming, see Attiya et al. [4] where each process identifier should be assigned to at most one process.

The at-most-once problem has also many similarities with the Write-All problem for the shared memory model [3, 9, 18, 23, 28, 36]. First presented by Kanellakis and Shvartsman [23], the Write-All problem is concerned with performing each job at-least-once. Most of the solutions for the Write-All problem, exhibit super-linear work even when $m \ll n$. Malewicz [36] was the first to present a solution for the Write-All problem that has linear work for a non-trivial number of processors. The algorithm presented by Malewicz [36] has work $\mathrm{O}\left(n+m^{4} \log n\right)$ and uses test-and-set operations. Later Kowalski and Shvartsman [28] presented a solution for the Write-All problem that for any constant $\epsilon$ has work $\mathrm{O}\left(n+m^{2+\epsilon}\right)$. Their algorithm uses a collection of $q$ permutations with contention $\mathrm{O}(q \log q)$ for a properly chosen constant $q$ and does not rely on test-and-set operations. Although an efficient polynomial time construction of permutations with contention $\mathrm{O}(q$ polylog $q)$ has been developed by Kowalski et al. [27], it is not known to date how to construct permutations with contention $\mathrm{O}(q \log q)$ in polynomial time. Subsequent to the conference version of this paper [25], Alistarh et al. [2] show that there exists a deterministic algorithm for the Write-All problem with work $\mathrm{O}\left(n+m \log ^{5} n \log ^{2} \max (n, m)\right)$, by derandomizing their randomized solution for the problem. Their solution is a breakthrough in terms of bridging the gap between the $\Omega(n+m \log m)$ lower bound for the Write-All problem and known deterministic solutions, but is so far existential. For a detailed overview of research on the Write-All problem, we refer the reader to the books by Georgiou and Shvartsman [15, 16].

We note that the at-most-once problem becomes much simpler when shared-memory is supplemented by some type of read-modify-write operations. For example, one can associate a test-and-set bit with each job, ensuring that the job is assigned to the only process that successfully sets the shared bit. An effectiveness optimal implementation can then be easily obtained from any Write-All solution. In this paper we deal only with the more challenging setting where algorithms use atomic read/write registers.

Contributions: We present and analyze the algorithm $\mathrm{KK}_{\beta}$ that solves the at-most-once problem. The algorithm is parametrized by $\beta \geq m$ and has effectiveness $n-\beta-m+2$. If $\beta<m$ the correctness of the algorithm is still guaranteed, but the termination of the algorithm cannot be guaranteed. For $\beta=m$ the algorithm has optimal effectiveness of $n-2 m+2$ up to an additive factor of $m$. Note that the upper bound for the effectiveness of any algorithm is $n-f$ [26], where $f \leq m-1$ is the number of failures in the system. We further prove that for $\beta \geq 3 m^{2}$ the algorithm has work complexity $\mathrm{O}(n m \log n \log m)$. We use algorithm $\mathrm{KK}_{\beta}$ with $\beta=3 m^{2}$, in order to construct an iterated version of our algorithm which for any constant $\epsilon>0$, has effectiveness of $n-\mathrm{O}\left(m^{2} \log n \log m\right)$ and work complexity $\mathrm{O}\left(n+m^{3+\epsilon} \log n\right)$. This is both effectiveness-optimal and work-optimal for any $m=\mathrm{O}(\sqrt[3+\epsilon]{n / \log n})$. We note that our solutions are deterministic and assume worst-case behavior. In the probabilistic setting CensorHillel [22] and Kentros et al. [24] show that optimal effectiveness can be achieved with expected work complexity $\mathrm{O}\left(n m^{2} \log m\right)$ and $\mathrm{O}\left(n+m^{2+\epsilon} \log n\right)$, for any small constant $\epsilon$, respectively.

We then demonstrate how to use the iterated version of our algorithm in order to solve the Write-All problem with work complexity $\mathrm{O}\left(n+m^{3+\epsilon} \log n\right)$ for any constant $\epsilon>0$. Our solution improves on the algorithm of Malewicz [36], which solves the Write-All problem for a non-trivial number of processes with optimal (linear) work complexity, in two ways. First our solution is work optimal for a wider range of choices for $m$, namely for any $m=\mathrm{O}(\sqrt[3+\epsilon]{n / \log n})$, cf. the restriction $m=\mathrm{O}(\sqrt[4]{n / \log n})$ of Malewicz, [36]. Second our solution does not assume the test-and-set primitive used by Malewicz and relies only on atomic read/write memory. There is 
also a Write-All algorithm due to Kowalski and Shvartsman [28], which does not use test-and-set operations and is work optimal for a wider range of processors $m$ than our algorithm, specifically for $m=\mathrm{O}(\sqrt[2+\epsilon]{n})$. However, their algorithm uses a collection of $q$ permutations with contention $\mathrm{O}(q \log q)$ and it is not known to date how to construct such permutations in polynomial time (see the discussion in the related work section). Finally, subsequent to the conference version of this paper [25], Alistarh et al. [2] show that there exists a deterministic algorithm for the Write-All problem with work $\mathrm{O}\left(n+m \log ^{5} n \log ^{2} \max (n, m)\right)$. Their solution is so far existential, while ours explicit.

Outline: In Section 2 we formalize the model and introduce definitions and notations used in the paper. In Section 3 we present the algorithm $\mathrm{KK}_{\beta}$. In Sections 4 and 5 we analyze correctness, effectiveness and work complexity of algorithm $\mathrm{KK}_{\beta}$. In Section 6 we present and analyze the iterative algorithm IterativeKK $(\epsilon)$. In Section7 we present and analyze the iterative algorithm WA_IterativeKK $(\epsilon)$ for the Write-All problem. Finally, we conclude with Section 8

\section{Model, Definitions, and Efficiency}

We define our model, the at-most-once problem, and measures of efficiency.

\subsection{Model and Adversary}

We model a multi-processor as $m$ asynchronous, crash-prone processes with unique identifiers from some set $\mathcal{P}$. Shared memory is modeled as a collection of atomic read/write memory cells, where the number of bits in each cell is explicitly defined. We use the Input/Output Automata formalism [34, 35] to specify and reason about algorithms; specifically, we use the asynchronous shared memory automaton formalization [17, 35]. Each process $p$ is defined in terms of its states states $_{p}$ and its actions acts $s_{p}$, where each action is of the type input, output, or internal. A subset start $_{p} \subseteq$ states $_{p}$ contains all the start states of $p$. Each shared variable $x$ takes values from a set $V_{x}$, among which there is $i_{n i t}$, the initial value of $x$.

We model an algorithm $A$ as a composition of the automata for each process $p$. Automaton $A$ consists of a set of states states $(A)$, where each state $s$ contains a state $s_{p} \in$ states $_{p}$ for each $p$, and a value $v \in V_{x}$ for each shared variable $x$. Start states $\operatorname{start}(A)$ is a subset of states $(A)$, where each state contains a $\operatorname{start}_{p}$ for each $p$ and an init $_{x}$ for each $x$. The actions of $A, \operatorname{acts}(A)$ consists of actions $\pi \in a c t s_{p}$ for each process $p$. A transition is the modification of the state as a result of an action and is represented by a triple $\left(s, \pi, s^{\prime}\right)$, where $s, s^{\prime} \in \operatorname{states}(A)$ and $\pi \in \operatorname{acts}(A)$. State $s$ is called the enabling state of action $\pi$. The set of all transitions is denoted by $\operatorname{trans}(A)$. Each action in $\operatorname{acts}(A)$ is performed by a process, thus for any transition $\left(s, \pi, s^{\prime}\right), s$ and $s^{\prime}$ may differ only with respect to the state $s_{p}$ of process $p$ that invoked $\pi$ and potentially the value of the shared variable that $p$ interacts with during $\pi$. We also use triples $\left(\left\{\operatorname{vars}_{s}\right\}, \pi,\left\{\right.\right.$ vars $\left.\left._{s^{\prime}}\right\}\right)$, where vars $_{s}$ and vars $_{s^{\prime}}$ are subsets of variables in $s$ and $s^{\prime}$ respectively, as a shorthand to describe transitions without having to specify $s$ and $s^{\prime}$ completely; here vars $s$ and vars $_{s^{\prime}}$ contain only the variables whose value changes as the result of $\pi$, plus possibly some other variables of interest.

An execution fragment of $A$ is either a finite sequence, $s_{0}, \pi_{1}, s_{1}, \ldots, \pi_{r}, s_{r}$, or an infinite sequence, $s_{0}, \pi_{1}, s_{1}, \ldots, \pi_{r}, s_{r}, \ldots$, of alternating states and actions, where $\left(s_{k}, \pi_{k+1}, s_{k+1}\right) \in$ $\operatorname{trans}(A)$ for any $k \geq 0$. If $s_{0} \in \operatorname{start}(A)$, then the sequence is called an execution. The set of executions of $A$ is $\operatorname{execs}(A)$. We say that execution $\alpha$ is fair, if $\alpha$ is finite and its last state is a state of $A$ where no locally controlled action is enabled, or $\alpha$ is infinite and every locally 
controlled action $\pi \in \operatorname{acts}(A)$ is performed infinitely many times or there are infinitely many states in $\alpha$ where $\pi$ is disabled. The set of fair executions of $A$ is fairexecs $(A)$. An execution fragment $\alpha^{\prime}$ extends a finite execution fragment $\alpha$ of $A$, if $\alpha^{\prime}$ begins with the last state of $\alpha$. We let $\alpha \cdot \alpha^{\prime}$ stand for the execution fragment resulting from concatenating $\alpha$ and $\alpha^{\prime}$ and removing the (duplicated) first state of $\alpha^{\prime}$.

For two states $s$ and $s^{\prime}$ of an execution fragment $\alpha$, we say that state $s$ precedes state $s^{\prime}$ and we write $s<s^{\prime}$ if $s$ appears before $s^{\prime}$ in $\alpha$. Moreover we write $s \leq s^{\prime}$ if state $s$ either precedes state $s^{\prime}$ in $\alpha$ or the states $s$ and $s^{\prime}$ are the same state of $\alpha$. We use the term precedes and the symbols $<$ and $\leq$ in a same way for the actions of an execution fragment. We use the term precedes and the symbol $<$ if an action $\pi$ appears before a state $s$ in an execution fragment $\alpha$ or if a state $s$ appears before an action $\pi$ in $\alpha$. Finally for a set of states $S$ of an execution fragment $\alpha$, we define as $s_{\max }=\max S$ the state $s_{\max } \in S$, s.t. $\forall s \in S, s \leq s_{\max }$ in $\alpha$.

We model process crashes by action $\operatorname{stop}_{p}$ in $\operatorname{acts}(A)$ for each process $p$. If stop appears $_{p}$ in an execution $\alpha$ then no actions $\pi \in$ acts $_{p}$ appear in $\alpha$ thereafter. We then say that process $p$ crashed. Actions stop $p$ arrive from some unspecified external environment, called an adversary. In this work we consider an omniscient, on-line adversary [23] that has complete knowledge of the algorithm executed by the processes. The adversary controls asynchrony and crashes. We allow up to $f<m$ crashes. We denote by fairexecs ${ }_{f}(A)$ all fair executions of $A$ with at most $f$ crashes. Note that since the processes can only communicate through atomic read/write operations in the shared memory, all the asynchronous executions are linearizable. This means that concurrent actions can be mapped to an equivalent sequence of state transitions, where only one process performs an action in each transition, and thus the model presented above is appropriate for the analysis of a multi-process asynchronous atomic read/write shared memory system.

\subsection{At-Most-Once Problem, Effectiveness and Complexity}

Let $A$ be an algorithm specified for $m$ processes with ids from set $\mathcal{P}=[1 \ldots m]$, and for $n$ jobs with unique ids from set $\mathcal{J}=[1 \ldots n]$. We assume that there are at least as many jobs as there are processes, i.e., $n \geq m$. We model the performance of job $j$ by process $p$ by means of action $\mathrm{do}_{p, j}$. For a sequence $c$, we let $\operatorname{len}(c)$ denote its length, and we let $\left.c\right|_{\pi}$ denote the sequence of elements $\pi$ occurring in $c$. Then for an execution $\alpha$, len $\left(\left.\alpha\right|_{\text {do }_{p, j}}\right)$ is the number of times process $p$ performs job $j$. Finally we denote by $F_{\alpha}=\left\{p \mid s t o p_{p}\right.$ occurs in $\left.\alpha\right\}$ the set of crashed processes in execution $\alpha$. Now we define the number of jobs performed in an execution. Note here that we are borrowing most definitions from Kentros et al. [26].

Definition 2.1. For execution $\alpha$ let $\mathcal{J}_{\alpha}=\left\{j \in \mathcal{J} \mid \mathrm{do}_{p, j}\right.$ occurs in $\alpha$ for some $\left.p \in \mathcal{P}\right\}$. The total number of jobs performed in $\alpha$ is defined to be $D o(\alpha)=\left|\mathcal{J}_{\alpha}\right|$.

We next define the at-most-once problem.

Definition 2.2. Algorithm $A$ solves the at-most-once problem if for each execution $\alpha$ of $A$ we have $\forall j \in \mathcal{J}: \sum_{p \in \mathcal{P}}$ len $\left(\left.\alpha\right|_{\mathrm{do}_{p, j}}\right) \leq 1$.

Definition 2.3. Let $S$ be a set of elements with unique identifiers. We define as the rank of element $x \in S$ and we write $[x]_{S}$, the rank of $x$ if we sort in ascending order the elements of $S$ according to their identifiers.

\section{Measures of Efficiency}

We analyze our algorithms in terms of two complexity measures: effectiveness and work. Effectiveness counts the number of jobs performed by an algorithm in the worst case. 
Definition 2.4. $E_{A}(n, m, f)=\min _{\alpha \in \text { fairexecs }_{f}(A)}(D o(\alpha))$ is the effectiveness of algorithm $A$, where $m$ is the number of processes, $n$ is the number of jobs, and $f$ is the number of crashes.

A trivial algorithm can solve the at-most-once problem by splitting the $n$ jobs in groups of size $\frac{n}{m}$ and assigning one group to each process. Such a solution has effectiveness $E(n, m, f)=$ $(m-f) \cdot \frac{n}{m}$ (consider an execution where $f$ processes fail at the beginning of the execution).

Work complexity measures the total number of basic operations (comparisons, additions, multiplications, shared memory reads and writes) performed by an algorithm. We assume that each internal or shared memory cell has size $\mathrm{O}(\log n)$ bits and performing operations involving a constant number of memory cell costs $\mathrm{O}(1)$. This is consistent with the way work complexity is measured in previous related work [23, 28, 36].

Definition 2.5. The work of algorithm $A$, denoted by $W_{A}$, is the worst case total number of basic operations performed by all the processes of algorithm $A$.

Finally we repeat here as a theorem, Corollary 1 from Kentros et al. [26], that gives an upper bound on the effectiveness for any algorithm solving the at-most-once problem.

Theorem 2.1. from Kentros et al. [26]

For all algorithms $A$ that solve the at-most-once problem with $m$ processes and $n \geq m$ jobs in the presence of $f<m$ crashes it holds that $E_{A}(n, m, f) \leq n-f$.

\section{Algorithm $\mathrm{KK}_{\beta}$}

We present algorithm $\mathrm{KK}_{\beta}$, that solves the at-most-once problem. Parameter $\beta \in \mathbb{N}$ is the termination parameter of the algorithm. Algorithm $\mathrm{KK}_{\beta}$ is defined for all $\beta \geq m$. If $\beta=m$, algorithm $\mathrm{KK}_{\beta}$ has optimal up to an additive factor of $m$ effectiveness. Note that although $\beta \geq m$ is not necessary in order to prove the correctness of the algorithm, if $\beta<m$ we cannot guarantee termination of algorithm $\mathrm{KK}_{\beta}$.

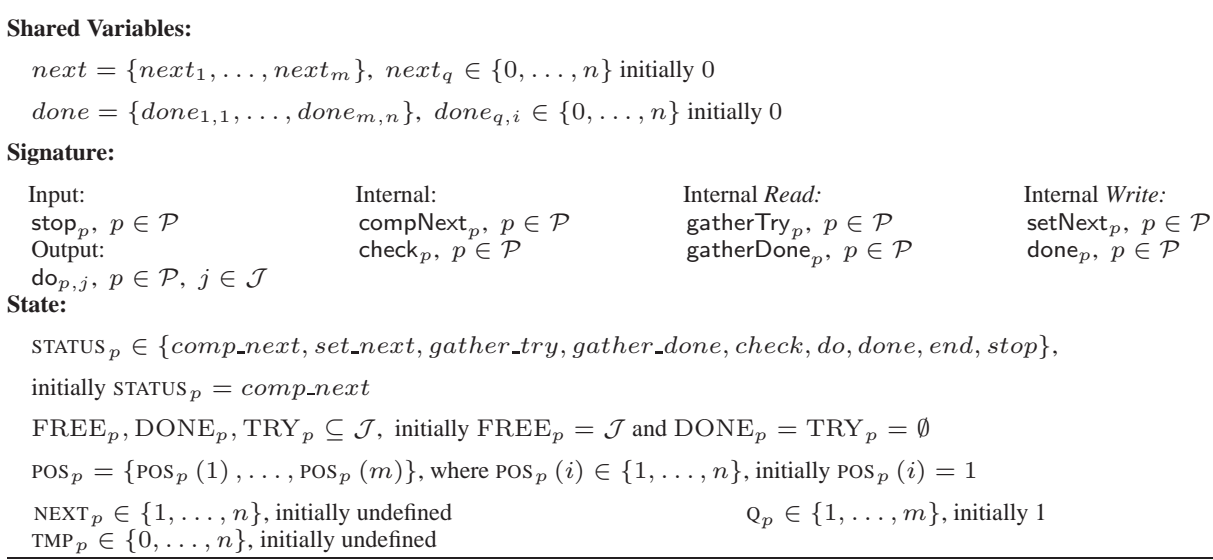

Figure 1: Algorithm $\mathrm{KK}_{\beta}$ : Shared Variables, Signature and States

The idea behind the algorithm $\mathrm{KK}_{\beta}$ (see Fig.11,2) is quite intuitive and is based on an algorithm for renaming processes presented by Attiya et al. [4]. Each process $p$, picks a job $i$ to perform, announces (by writing in shared memory) that it is about to perform the job and then 
checks if it is safe to perform it (by reading the announcements other processes made in the shared memory, and the jobs other processes announced they have performed). If it is safe to perform the job $i$, process $p$ will proceed with the $\mathrm{do}_{p, i}$ action and then mark the job completed. If it is not safe to perform $i, p$ will release the job. In either case, $p$ picks a new job to perform. In order to pick a new job, $p$ reads from the shared memory and gathers information on which jobs are safe to perform, by reading the announcements that other processes made in the shared memory about the jobs they are about to perform, and the jobs other processes announced they have already performed. Assuming that those jobs are ordered, $p$ splits the set of "free" jobs in $m$ intervals and picks the first job of the interval with rank equal to $p$ 's rank. Note that since the information needed in order to decide whether it is safe to perform a specific job and in order to pick the next job to perform is the same, these steps are combined in the algorithm. In Figure 2 we use function $\operatorname{rank}\left(\mathrm{SET}_{1}, \mathrm{SET}_{2}, i\right)$, that returns the element of set $\mathrm{SET}_{1} \backslash \mathrm{SET}_{2}$ that has rank $i$. If $\mathrm{SET}_{1}$ and $\mathrm{SET}_{2}$ have $\mathrm{O}(n)$ elements and are stored in some tree structure like redblack tree or some variant of $B$-tree, the operation $\operatorname{rank}\left(\mathrm{SET}_{1}, \mathrm{SET}_{2}, i\right)$, costs $\mathrm{O}\left(\left|\mathrm{SET}_{2}\right| \log n\right)$ assuming that $\mathrm{SET}_{2} \subseteq \mathrm{SET}_{1}$.

We will prove that algorithm $\mathrm{KK}_{\beta}$ has effectiveness $n-(\beta+m-2)$. For $\beta=O(m)$ this effectiveness is asymptotically optimal for any $m=o(n)$. Note that by Theorem 2.1 the upper bound on effectiveness of the at-most-once problem is $n-f$, where $f$ is the number of failed processes in the system. Next we present algorithm $\mathrm{KK}_{\beta}$ in more detail.

Shared Variables. next is an array with $m$ elements. In the cell next $t_{q}$ of the array process $q$ announces the job it is about to perform. From the structure of algorithm $\mathrm{KK}_{\beta}$, only process $q$ writes in cell next $t_{q}$. On the other hand any process may read cell next $t_{q}$.

done is an $m \times n$ matrix. In line $q$ of the matrix, process $q$ announces the jobs it has performed. Each cell of line $q$ contains the identifier of exactly one job that has been performed by process $q$. Only process $q$ writes in the cells of line $q$ but any process may read them. Moreover, process $q$ updates line $q$ by adding entries at the end of it.

Internal Variables of process $p$. The variable $\operatorname{STATUS}_{p}$ records the status of process $p$ and defines its next action as follows: STATUS $_{p}=$ comp_next - process $p$ is ready to compute the next job to perform (this is the initial status of $p$ ), STATUS $_{p}=$ set_next - $p$ computed the next job to perform and is ready to announce it by writing in the shared memory, STATUS $_{p}=$ gather_try - $p$ reads the array next in shared memory in order to compute the $\mathrm{TRY}_{p}$ set, STATUS $_{p}=$ gather_done - $p$ reads the matrix done in shared memory in order to update the $\mathrm{DONE}_{p}$ and $\mathrm{FREE}_{p}$ sets, STATUS $_{p}=$ check $-p$ has to check whether it is safe to perform its current job, STATUS $_{p}=d o-p$ can safely perform its current job, STATUS $_{p}=d o n e-p$ performed its current job and needs to update the shared memory, STATUS $_{p}=e n d-p$ terminated, STATUS $_{p}=$ stop $p$ crashed.

$\mathrm{FREE}_{p}, \mathrm{DONE}_{p}, \mathrm{TRY}_{p} \subseteq \mathcal{J}$ are three sets that are used by process $p$ in order to compute the next job to perform and whether it is safe to perform it. We use some tree structure like redblack tree or some variant of $B$-tree [5, 19] for the sets $\mathrm{FREE}_{p}, \mathrm{DONE}_{p}$ and $\mathrm{TRY}_{p}$, in order to be able to add, remove and search elements in them with $\mathrm{O}(\log n)$ work. $\mathrm{FREE}_{p}$, is initially set to $\mathcal{J}$ and contains an estimate of the jobs that are still available. $\mathrm{DONE}_{p}$ is initially empty and contains an estimate of the jobs that have been performed. No job is removed from $\mathrm{DONE}_{p}$ or added to $\mathrm{FREE}_{p}$ during the execution of algorithm $\mathrm{KK}_{\beta} \cdot \mathrm{TRY}_{p}$ is initially empty and contains an estimate of the jobs that other processes are about to perform. It holds that $\left|\mathrm{TRY}_{p}\right|<m$, since there are $m-1$ processes apart from process $p$ that may be attempting to perform a job.

$\operatorname{POS}_{p}$ is an array of $m$ elements. Position $\operatorname{POS}_{p}(q)$ of the array contains a pointer in the line 


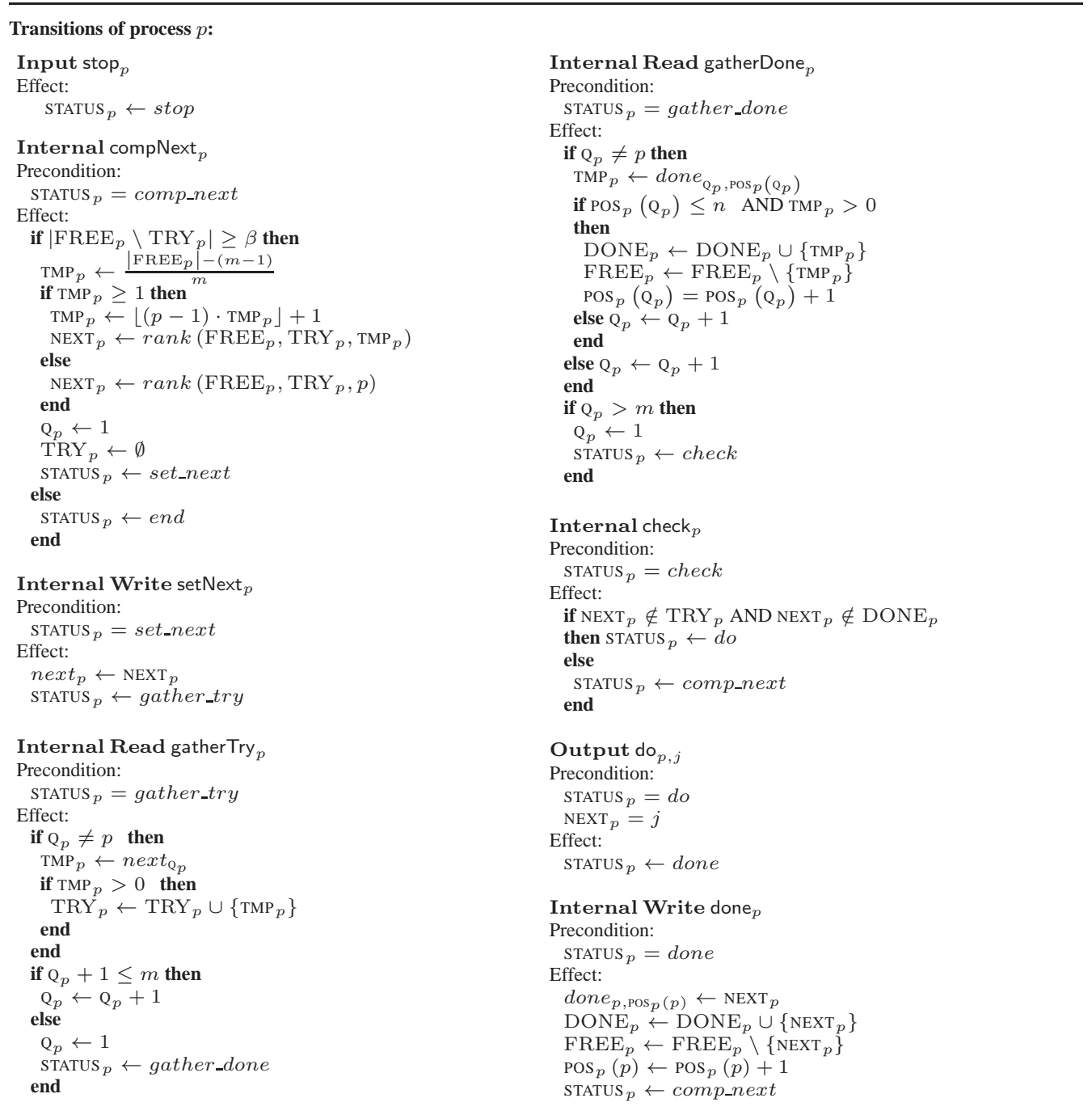

Figure 2: Algorithm $\mathrm{KK}_{\beta}$ : Transitions

$q$ of the shared matrix done. $\operatorname{POS}_{p}(q)$ is the element of line $q$ that process $p$ will read from. In the special case where $q=p, \operatorname{POS}_{p}(p)$ is the element of line $p$ that process $p$ will write into after performing a new job. The elements of the shared matrix done are read when process $p$ is updating the $\mathrm{DONE}_{p}$ set.

$\mathrm{NEXT}_{p}$ contains the job process $p$ is attempting to perform.

$\mathrm{TMP}_{p}$ is a temporary storage for values read from the shared memory.

$\mathrm{Q}_{p} \in\{1, \ldots, m\}$ is used as indexing for looping through process identifiers.

Actions of process $p$. We visit them one by one below.

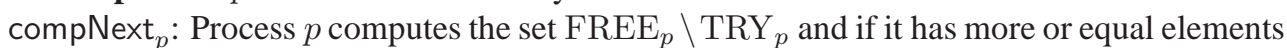
to $\beta$, were $\beta$ is the termination parameter of the algorithm, process $p$ computes its next candidate job, by splitting the $\mathrm{FREE}_{p} \backslash \mathrm{TRY}_{p}$ set in $m$ parts and picking the first element of the $p$-th 
part. In order to do that it uses the function $\operatorname{rank}\left(\mathrm{SET}_{1}, \mathrm{SET}_{2}, i\right)$, which returns the element of set $\mathrm{SET}_{1} \backslash \mathrm{SET}_{2}$ with rank $i$. Finally process $p$ sets the $\mathrm{TRY}_{p}$ set to the empty set, the $\mathrm{Q}_{p}$ internal variable to 1 and its status to set_next in order to update the shared memory with its new candidate job. If the $\mathrm{FREE}_{p} \backslash \mathrm{TRY}_{p}$ set has less than $\beta$ elements process $p$ terminates.

setNext ${ }_{p}$ : Process $p$ announces its new candidate job by writing the contents of its $\mathrm{NEXT}_{p}$ internal variable in the $p$-th position of the next array. Remember that the next array is stored in shared memory. Process $p$ changes its status to gather_try, in order to start collecting the $\mathrm{TRY}_{p}$ set from the next array.

gatherTry $_{p}$ : With this action process $p$ implements a loop, which reads from the shared memory all the positions of the array next and updates the $\mathrm{TRY}_{p}$ set. In each execution of the action, process $p$ checks if $\mathrm{Q}_{p}$ is equal to $p$. If it is not equal, $p$ reads the $\mathrm{Q}_{p}$-th position of the array next, checks if the value read is greater than 0 and if it is, adds the value it read in the $\mathrm{TRY}_{p}$ set. If $\mathrm{Q}_{p}$ is equal to $p, p$ just skips the step described above. Then $p$ checks if the value of $\mathrm{Q}_{p}+1$ is less than $m+1$. If it is, then $p$ increases $\mathrm{Q}_{p}$ by 1 and leaves its status gather_try, otherwise $p$ has finished updating the $\mathrm{TRY}_{p}$ set and thus sets $\mathrm{Q}_{p}$ to 1 and changes its status to gather_done, in order to update the $\mathrm{DONE}_{p}$ and $\mathrm{FREE}_{p}$ sets from the contents of the done matrix.

gatherDone ${ }_{p}$ : With this action process $p$ implements a loop, which updates the $\mathrm{DONE}_{p}$ and $\mathrm{FREE}_{p}$ sets with values read from the matrix done, which is stored in shared memory. In each execution of the action, process $p$ checks if $\mathrm{Q}_{p}$ is equal to $p$. If it is not equal, $p$ uses the internal variable $\operatorname{POS}_{p}\left(\mathrm{Q}_{p}\right)$, in order to read fresh values from the line $\mathrm{Q}_{p}$ of the done matrix. In detail, $p$ reads the shared variable done $e_{\mathrm{Q}_{p}, \operatorname{Pos}_{p}\left(\mathrm{Q}_{p}\right)}$, checks if $\operatorname{POS}_{p}\left(\mathrm{Q}_{p}\right)$ is less than $n+1$ and if the value read is greater than 0 . If both conditions hold, $p$ adds the value read at the $\mathrm{DONE}_{p}$ set, removes the value read from the $\mathrm{FREE}_{p}$ set and increases $\operatorname{POS}_{p}\left(\mathrm{Q}_{p}\right)$ by one. Otherwise, it means that either process $\mathrm{Q}_{p}$ has terminated (by performing all the $n$ jobs) or the line $\mathrm{Q}_{p}$ does not contain any new completed jobs. In either case $p$ increases the value of $\mathrm{Q}_{p}$ by 1 . The value of $\mathrm{Q}_{p}$ is increased by 1 also if $\mathrm{Q}_{p}$ was equal to $p$. Finally $p$ checks whether $\mathrm{Q}_{p}$ is greater than $m$; if it is, $p$ has completed the loop and thus changes its status to check.

check $_{p}$ : Process $p$ checks if it is safe to perform its current job. This is done by checking if $\mathrm{NEXT}_{p}$ belongs to the set $\mathrm{TRY}_{p}$ or to the set $\mathrm{DONE}_{p}$. If it does not, then it is safe to perform the job $\operatorname{NEXT}_{p}$ and $p$ changes its status to $d o$. Otherwise it is not safe, and thus $p$ changes its status to comp_next, in order to find a new job that may be safe to perform.

$\mathrm{do}_{p, j}$ : Process $p$ performs job $j$. Note that $\operatorname{NEXT}_{p}=j$ is part of the preconditions for the action to be enabled in a state. Then $p$ changes its status to done.

done $_{p}$ : Process $p$ writes in the done $e_{p, \operatorname{Pos}_{p}(p)}$ position of the shared memory the value of $\operatorname{NEXT}_{p}$, letting other processes know that it performed job $\operatorname{NEXT}_{p}$. Also $p$ adds $\operatorname{NEXT}_{p}$ to its $\mathrm{DONE}_{p}$ set, removes NEXT $p$ from its $\mathrm{FREE}_{p}$ set, increases $\operatorname{POS}_{p}(p)$ by 1 and changes its status to $c o m p \_n e x t$.

stop $_{p}$ : Process $p$ crashes by setting its status to stop.

\section{Correctness and Effectiveness Analysis}

We begin the analysis of algorithm $\mathrm{KK}_{\beta}$, by showing in Lemma 4.1 that $\mathrm{KK}_{\beta}$ solves the at-most-once problem. That is, there exists no execution of $\mathrm{KK}_{\beta}$ in which 2 distinct actions $\mathrm{do}_{p, i}$ and $\mathrm{do}_{q, i}$ appear for some $i \in \mathcal{J}$ and $p, q \in \mathcal{P}$. We continue the analysis by showing in Theorem 4.4 that algorithm $\mathrm{KK}_{\beta}$ has effectiveness $E_{\mathrm{KK}_{\beta}}(n, m, f)=n-(\beta+m-2)$. This is 
done in two steps. First in Lemma 4.2 we show that algorithm $\mathrm{KK}_{\beta}$ cannot terminate its execution if less than $n-(\beta+m-1)$ jobs are performed. The effectiveness analysis is completed by showing in Lemma 4.3 that the algorithm is wait-free (it has no infinite fair executions). In Theorem 4.4 we combine the two lemmas in order to show that the effectiveness of algorithm $\mathrm{KK}_{\beta}$ is greater that or equal to $n-(\beta+m-2)$. Moreover, we show the existence of an adversarial strategy, that results in a terminating execution where $n-(\beta+m-2)$ jobs are completed, showing that the bound is tight.

In the analysis that follows, for a state $s$ and a process $p$ we denote by $s . \mathrm{FREE}_{p}, s . \mathrm{DONE}_{p}, s . \mathrm{TRY}_{p}$, the values of the internal variables FREE, DONE and TRY of process $p$ in state $s$. Moreover with s.next, and s.done we denote the contents of the array next and the matrix done in state $s$. Remember that next and done, are stored in shared memory.

Lemma 4.1. There exists no execution $\alpha$ of algorithm $\mathrm{KK}_{\beta}$, such that $\exists i \in \mathcal{J}$ and $\exists p, q \in \mathcal{P}$ for which $\mathrm{do}_{p, i}, \mathrm{do}_{q, i} \in \alpha$.

Proof. Let us for the sake of contradiction assume that there exists an execution $\alpha \in$ $\operatorname{execs}\left(\mathrm{KK}_{\beta}\right)$ and $i \in \mathcal{J}$ and $p, q \in \mathcal{P}$ such that $\mathrm{do}_{p, i}, \mathrm{do}_{q, i} \in \alpha$. We examine two cases.

Case $1 p=q$ : Let states $s_{1}, s_{1}^{\prime}, s_{2}, s_{2}^{\prime} \in \alpha$, such that the transitions $\left(s_{1}, \mathrm{do}_{p, i}, s_{1}^{\prime}\right)$, $\left(s_{2}, \mathrm{do}_{p, i}, s_{2}^{\prime}\right) \in \alpha$ and without loss of generality assume $s_{1}^{\prime} \leq s_{2}$ in $\alpha$. From Figure 2 we have that $s_{1}^{\prime} \cdot \operatorname{NEXT}_{p}=i, s_{1}^{\prime} \cdot \operatorname{STATUS}_{p}=d o n e$ and $s_{2} \cdot \mathrm{NEXT}_{p}=i, s_{2} \cdot \mathrm{STATUS}_{p}=d o$. From algorithm $\mathrm{KK}_{\beta}$, state $s_{2}$ must be preceded by transition $\left(s_{3}\right.$, check $\left._{p}, s_{3}^{\prime}\right)$, such that $s_{3} \cdot \mathrm{NEXT}_{p}=i$ and $s_{3}^{\prime} \cdot \operatorname{NEXT}_{p}=i, s_{3}^{\prime} \cdot \operatorname{STATUS}_{p}=d o$, where $s_{1}^{\prime}$ precedes $s_{3}$ in $\alpha$. Finally $s_{3}$ must be preceded in $\alpha$ by transition $\left(s_{4}\right.$, done $\left.e_{p}, s_{4}^{\prime}\right)$, where $s_{1}^{\prime}$ precedes $s_{4}$, such that $s_{4} \cdot \mathrm{NEXT}_{p}=i$ and $i \in s_{4}^{\prime}$. DONE $E_{p}$. Since $s_{4}^{\prime}$ precedes $s_{3}$ and during the execution of $\mathrm{KK}_{\beta}$ no elements are removed from $\mathrm{DONE}_{p}$, we have that $i \in s_{3} \cdot \mathrm{DONE}_{p}$. This is a contradiction, since the transition $\left(\left\{\operatorname{NEXT}_{p}=i, i \in \operatorname{DONE}_{p}\right\}, \operatorname{check}_{p},\left\{\operatorname{NEXT}_{p}=i, \operatorname{STATUS}_{p}=d o\right\}\right) \notin \operatorname{trans}\left(\mathrm{KK}_{\beta}\right)$.

Case $2 p \neq q$ : Given transition $\left(s_{1}, \mathrm{do}_{p, i}, s_{1}^{\prime}\right)$ in execution $\alpha$, we deduce from Fig.2 that there exist in $\alpha$ transitions $\left(s_{2}, \operatorname{setNext}_{p}, s_{2}^{\prime}\right),\left(s_{3}\right.$, gatherTry $\left._{p}, s_{3}^{\prime}\right),\left(s_{4}\right.$, check $\left._{p}, s_{4}^{\prime}\right)$, where $s_{2}^{\prime} \cdot$ next $_{p}=s_{2}^{\prime} \cdot \mathrm{NEXT}_{p}=i, s_{3} \cdot$ next $_{p}=s_{3} \cdot \mathrm{NEXT}_{p}=i, s_{3} \cdot \mathrm{Q}_{p}=q, s_{4} \cdot \mathrm{NEXT}_{p}=i, s_{4}^{\prime} \cdot \mathrm{NEXT}_{p}=$ $i, s_{4}^{\prime}$. STATUS $_{p}=d o$, such that $s_{2}<s_{3}<s_{4}<s_{1}$ and there exists no action $\pi=$ compNext $_{p}$ in execution $\alpha$, such that $s_{2}<\pi<s_{1}^{\prime}$.

Similarly for transition $\left(t_{1}, \mathrm{do}_{q, i}, t_{1}^{\prime}\right)$ there exist in execution $\alpha$ transitions $\left(t_{2}, \operatorname{setNext}_{q}, t_{2}^{\prime}\right),\left(t_{3}\right.$, gatherTry $\left._{q}, t_{3}^{\prime}\right),\left(t_{4}\right.$, check $\left._{q}, t_{4}^{\prime}\right)$, where $t_{2}^{\prime} \cdot$ next $_{q}=t_{2}^{\prime} \cdot \mathrm{NEXT}_{q}=i$, $t_{3} \cdot$ next $_{q}=t_{3} \cdot \mathrm{NEXT}_{q}=i, t_{3} \cdot \mathrm{Q}_{q}=p, t_{4} \cdot \mathrm{NEXT}_{q}=i, t_{4}^{\prime} \cdot \mathrm{NEXT}_{q}=i, t_{4}^{\prime} \cdot \mathrm{STATUS}_{q}=d o$, such that $t_{2}<t_{3}<t_{4}<t_{1}$ and there exists no action $\pi^{\prime}=$ compNext $_{q}$ in execution $\alpha$, such that $t_{2}<\pi<t_{1}^{\prime}$.

Either state $s_{2}<t_{3}$ or $t_{3}<s_{2}$ which implies $t_{2}<s_{3}$. We will show that if $s_{2}<t_{3}$ then $\mathrm{do}_{q, i}$ cannot take place, leading to a contradiction. The case where $t_{2}<s_{3}$ is symmetric and will be omitted.

Let us assume that $s_{2}$ precedes $t_{3}$. We have two cases, either $t_{3}$.next $t_{p}=i$ or $t_{3}$.next $t_{p} \neq i$.

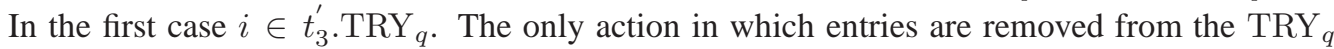




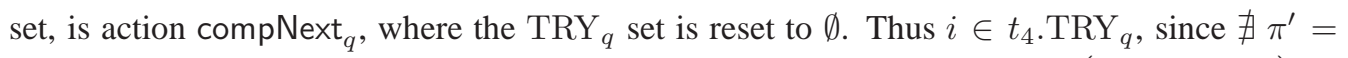
compNext $_{q} \in \alpha$, such that $t_{2}<\pi^{\prime}<t_{1}$. This is a contradiction since $\left(t_{4}\right.$, check $\left._{q}, t_{4}^{\prime}\right) \notin$ $\operatorname{trans}\left(\mathrm{KK}_{\beta}\right)$, if $i \in t_{4} \cdot \mathrm{TRY}_{q}, t_{4} \cdot \mathrm{NEXT}_{q}=i$ and $t_{4}^{\prime} \cdot \operatorname{STATUS}_{q}=d o$.

If $t_{3}$.next $t_{p} \neq i$, since $\left(s_{2}, \operatorname{setNext}_{p}, s_{2}^{\prime}\right) \in \alpha$ and $s_{2}^{\prime}<t_{3}$ there exists action $\pi_{1}=$ setNext $t_{p} \in \alpha$, such that $s_{2}^{\prime}<\pi_{1}<t_{3}$. Moreover, there exists action $\pi_{2}=\operatorname{compNext}_{p}$ in $\alpha$, such that $s_{2}^{\prime}<\pi_{2}<\pi_{1}$. Since $\nexists \pi=$ compNext $_{p} \in \alpha$, such that $s_{2}<\pi<s_{1}^{\prime}$, it holds that $s_{1}^{\prime}<\pi_{2}<\pi_{1}<t_{3}$. Furthermore, from Fig.2 there exists transition $\left(s_{5}\right.$, done $\left._{p}, s_{5}^{\prime}\right)$ in $\alpha$ and $j \in\{1, \ldots, n\}$, such that $s_{5} \cdot \operatorname{POS}_{p}(p)=j, s_{5} \cdot$ done $_{p, j}=0, s_{5} \cdot \mathrm{NEXT}_{p}=i, s_{5}^{\prime} \cdot$ done $_{p, j}=i$ and $s_{1}^{\prime}<s_{5}^{\prime}<\pi_{2}<t_{3}$. It must be the case that $i \notin t_{2} . D O N E_{q}$, since $t_{2} \cdot \mathrm{NEXT}_{q}=i$. From that and from Fig. 22 we have that there exists transition $\left(t_{6}\right.$, gatherDone $\left.{ }_{q}, t_{6}^{\prime}\right)$ in $\alpha$, such that $t_{6} \cdot \mathrm{Q}_{q}=p, t_{6} \cdot \operatorname{POS}_{q}(p)=j$ and $t_{3}<t_{6}<t_{4}$. Since $s_{5}^{\prime}<t_{3}$ and done $e_{p, j}$ from algorithm $\mathrm{KK}_{\beta}$ cannot be changed again in execution $\alpha$, we have that $t_{6} \cdot$ done $_{p, j}=i$ and as a result $i \in t_{6}^{\prime}$. $\mathrm{DONE}_{q}$. Moreover, during the execution of algorithm $\mathrm{KK}_{\beta}$, entries in set $\mathrm{DONE}_{q}$ are only added and never removed, thus we have that $i \in t_{4} . D O N E_{q}$. This is a contradiction since $\left(t_{4}, \operatorname{check}_{q}, t_{4}^{\prime}\right) \notin \operatorname{trans}\left(\mathrm{KK}_{\beta}\right)$, if $i \in t_{4} \cdot \mathrm{DONE}_{q}, t_{4} \cdot \mathrm{NEXT}_{q}=i$ and $t_{4}^{\prime} \cdot \mathrm{STATUS}_{q}=d o$. This completes the proof.

Next we examine the effectiveness of the algorithm. First we show that algorithm $\mathrm{KK}_{\beta}$ cannot terminate its execution if less than $n-(\beta+m-1)$ jobs are performed.

Lemma 4.2. For any $\beta \geq m, f \leq m-1$ and for any finite execution $\alpha \in$ execs $\left(\mathrm{KK}_{\beta}\right)$ with $D o(\alpha) \leq n-(\beta+m-1)$, there exists a (non-empty) execution fragment $\alpha^{\prime}$ such that $\alpha \cdot \alpha^{\prime} \in \operatorname{execs}\left(\mathrm{KK}_{\beta}\right)$.

Proof. From the algorithm $\mathrm{KK}_{\beta}$, we have that for any process $p$ and any state $s \in \alpha$, $\left|s \cdot \mathrm{FREE}_{p}\right| \geq n-D o(\alpha)$ and $\left|s . \mathrm{TRY}_{p}\right| \leq m-1$. The first inequality holds since the $s . \mathrm{FREE}_{p}$ set is estimated by $p$ by examining the done matrix which is stored in shared memory. From algorithm $\mathrm{KK}_{\beta}$, a job $j$ is only inserted in line $q$ of the matrix done, if a do $q, j$ action has already been performed by process $q$. The second inequality is obvious. Thus we have that $\forall p \in \mathcal{P}$ and $\forall s \in \alpha,\left|s \cdot \mathrm{FREE}_{p} \backslash s \cdot \mathrm{TRY}_{p}\right| \geq n-(D o(\alpha)+m-1)$. If $D o(\alpha) \leq n-(\beta+m-1)$, $\forall p \in \mathcal{P}$ and $\forall s \in \alpha$ we have that $\left|s . \mathrm{FREE}_{p} \backslash s . \mathrm{TRY}_{p}\right| \geq \beta$. Since there can be $f \leq m-1$ failed processes in our system, at the final state $s^{\prime}$ of execution $\alpha$ there exists at least one process $p \in \mathcal{P}$ that has not failed. This process has not terminated, since from Fig. 2 a process $p$ can only terminate if in the enabling state $s$ of action compNext $p,\left|s \cdot \mathrm{FREE}_{p} \backslash s \cdot \mathrm{TRY}_{p}\right|<\beta$. This process can continue executing steps and thus there exists a (non-empty) execution fragment $\alpha^{\prime}$ such that $\alpha \cdot \alpha^{\prime} \in \operatorname{execs}\left(\mathrm{KK}_{\beta}\right)$.

Since no finite execution of algorithm $\mathrm{KK}_{\beta}$ can terminate if less than $n-(\beta+m-1)$ jobs are performed, Lemma4.2 implies that if the algorithm $\mathrm{KK}_{\beta}$ has effectiveness less than or equal to $n-(\beta+m-1)$, there must exist some infinite fair execution $\alpha$ with $D o(\alpha) \leq n-(\beta+m-1)$. Next we prove that algorithm $\mathrm{KK}_{\beta}$ is wait-free (it has no infinite fair executions).

Lemma 4.3. For any $\beta \geq m, f \leq m-1$ there exists no infinite fair execution $\alpha \in \operatorname{execs}\left(\mathrm{KK}_{\beta}\right)$. 
Proof. We will prove this by contradiction. Let $\beta \geq m$ and $\alpha \in \operatorname{execs}\left(\mathrm{KK}_{\beta}\right)$ an infinite fair execution with $f \leq m-1$ failures, and let $D o(\alpha)$ be the jobs executed by execution $\alpha$ according to Definition2.1 Since $\alpha \in \operatorname{execs}\left(\mathrm{KK}_{\beta}\right)$ and from Lemma $4.1 \mathrm{KK}_{\beta}$ solves the at-most-once problem, $D o(\alpha)$ is finite. Clearly there exists at least one process in execution $\alpha$ that has not crashed and does not terminate (some process must take steps in $\alpha$ in order for it to be infinite). Since $D o(\alpha)$ and $f$ are finite, there exists a state $s_{0}$ in $\alpha$ such that after $s_{0}$ no process crashes, no process terminates, no do action takes place in $\alpha$ and no process adds new entries in the done matrix in shared memory. The later holds since the execution is infinite and fair, the $D o(\alpha)$ is also finite, consequently any non failed process $q$ that has not terminated will eventually update the $q$ line of the done matrix to be in agreement with the $\mathrm{do}_{q, *}$ actions it has performed. Moreover any process $q$ that has terminated, has already updated the $q$ line of done matrix with the latest do action it performed, before it terminated, since in order to terminate it must have reached a compNext action that has set its status to end.

We define the following sets of processes and jobs according to state $s_{0}$. $\mathcal{J}_{\alpha}$ are jobs that have been performed in $\alpha$ according to Definition 2.1 $\mathcal{P}_{\alpha}$ are processes that do not crash and do not terminate in $\alpha$. By the way we defined state $s_{0}$ only processes in $\mathcal{P}_{\alpha}$ take steps in $\alpha$ after state $s_{0}$. STUCK ST $_{\alpha}=\left\{i \in \mathcal{J} \backslash \mathcal{J}_{\alpha} \mid \exists\right.$ failed process $p: s_{0}$. next $\left.t_{p}=i\right\}$, i.e., STUCK $_{\alpha}$ expresses the set of jobs that are held by failed processes. DONE $_{\alpha}=$ $\left\{i \in \mathcal{J}_{\alpha} \mid \exists p \in \mathcal{P}\right.$ and $j \in\{1, \ldots, n\}: s_{0}$.done $\left.e_{p}(j)=i\right\}$, i.e., $\mathrm{DONE}_{\alpha}$ expresses the set of jobs that have been performed before state $s_{0}$ and the processes that performed them managed to update the shared memory. Finally we define $\mathrm{POOL}_{\alpha}=\mathcal{J} \backslash\left(\mathcal{J}_{\alpha} \cup \mathrm{STUCK}_{\alpha}\right)$. After state $s_{0}$, all processes in $\mathcal{P}_{\alpha}$ will keep executing. This means that whenever a process $p \in \mathcal{P}_{\alpha}$ takes action compNext $_{p}$ in $\alpha$, the first if statement is true. Specifically it holds that for $\forall p \in \mathcal{P}_{\alpha}$ and for all the enabling states $s \geq s_{0}$ of actions compNext in $\alpha,\left|\mathrm{FREE}_{p} \backslash \mathrm{TRY}_{p}\right| \geq \beta$.

From Figure 2 we have that for any $p \in \mathcal{P}_{\alpha}, \exists s_{p} \in \alpha$ such that $s_{p}>s_{0}$ and for all states $s \geq s_{p}, s \cdot \mathrm{DONE}_{p}=\mathrm{DONE}_{\alpha}, s \cdot \mathrm{FREE}_{p}=\mathcal{J} \backslash \mathrm{DONE}_{\alpha}$ and $s \cdot \mathrm{FREE}_{p} \backslash s \cdot \mathrm{TRY}_{p} \subseteq \mathrm{POOL}_{\alpha}$. Let $s_{0}^{\prime}=\max _{p \in \mathcal{P}_{\alpha}}\left[s_{p}\right]$. From the above we have: $\left|\mathcal{J} \backslash \mathrm{DONE}_{\alpha}\right| \geq \beta \geq m$ and $\left|\mathrm{POOL}_{\alpha}\right| \geq$ $\beta \geq m$, since $\forall s^{\prime} \geq s_{0}^{\prime}$ we have that $s^{\prime} . \mathrm{FREE}_{p}=\mathcal{J} \backslash \mathrm{DONE}_{\alpha}$ and $s^{\prime} . \mathrm{FREE}_{p} \backslash s^{\prime} . \mathrm{TRY}_{p} \subseteq$ $\mathrm{POOL}_{\alpha}$ and $\forall p \in \mathcal{P}_{\alpha}$ and for all the enabling states $s \geq s_{0}^{\prime}$ of actions compNext ${ }_{p}$ in $\alpha$, we have that $\left|\mathrm{FREE}_{p} \backslash \mathrm{TRY}_{p}\right| \geq \beta$.

Let $p_{0}$ be the process with the smallest process identifier in $\mathcal{P}_{\alpha}$. We examine 2 cases according to the size of $\mathcal{J} \backslash \mathrm{DONE}_{\alpha}$.

Case $\mathbf{A}\left|\mathcal{J} \backslash \mathrm{DONE}_{\alpha}\right| \geq 2 m-1$ : Let $x_{0} \in \mathrm{POOL}_{\alpha}$ be the job such that $\left[x_{0}\right]_{\mathrm{POOL}_{\alpha}}=$ $\left\lfloor\left(p_{0}-1\right) \cdot \frac{\left|\mathcal{J} \backslash \mathrm{DONE}_{\alpha}\right|-(m-1)}{m}\right\rfloor+1$. Such $x_{0}$ exists since $\forall p \in \mathcal{P}_{\alpha}$ and $\forall s \geq s_{0}^{\prime}$ it holds $s . \mathrm{FREE}_{p} \backslash s \cdot \mathrm{TRY}_{p} \subseteq \mathrm{POOL}_{\alpha}, s . \mathrm{FREE}_{p}=\mathcal{J} \backslash \mathrm{DONE}_{\alpha}$ from which we have that $\left|\mathrm{POOL}_{\alpha}\right| \geq$ $\left|\mathcal{J} \backslash \mathrm{DONE}_{\alpha}\right|-\left|s \cdot \mathrm{TRY}_{p}\right| \geq\left|\mathcal{J} \backslash \mathrm{DONE}_{\alpha}\right|-(m-1) \geq m$.

It follows that any $p \in \mathcal{P}_{\alpha}$ that executes action compNext $t_{p}$ after state $s_{0}^{\prime}$, will have its $\operatorname{NEXT}_{p}$ variable pointing in a job $x$ with $[x]_{\mathrm{POOL}_{\alpha}} \geq\left\lfloor(p-1) \cdot \frac{\left|\mathcal{J} \backslash \mathrm{DONE}_{\alpha}\right|-(m-1)}{m}\right\rfloor+1$. Thus $\forall p \in \mathcal{P}_{\alpha}$, $\exists s_{p}^{\prime} \geq s_{0}^{\prime}$ in $\alpha$ such that $\forall$ states $s \geq s_{p}^{\prime},\left[\text { s.next }_{p}\right]_{\mathrm{POOL}_{\alpha}} \geq\left\lfloor(p-1) \cdot \frac{\left|\mathcal{J} \backslash \mathrm{DONE}_{\alpha}\right|-(m-1)}{m}\right\rfloor+1$. Let $s_{0}^{\prime \prime}=\max _{p \in \mathcal{P}_{\alpha}}\left[s_{p}^{\prime}\right]$, we have 2 cases for $p_{0}$ :

Case A.1) After $s_{0}^{\prime \prime}$, process $p_{0}$ executes action compNext $p_{0}$ and the transition leads to state $s_{1}>s_{0}^{\prime \prime}$ such that $s_{1} \cdot \mathrm{NEXT}_{p_{0}}=x_{0}$. Since $\left[x_{0}\right]_{\mathrm{POOL}_{\alpha}}=\left\lfloor\left(p_{0}-1\right) \cdot \frac{\left|\mathcal{J} \backslash \mathrm{DONE}_{\alpha}\right|-(m-1)}{m}\right\rfloor+1$ and $p_{0}=\min _{p \in \mathcal{P}_{\alpha}}[p]$, from the previous discussion we have that $\forall s \geq s_{1}$ and $\forall p \in \mathcal{P} \backslash\left\{p_{0}\right\}$, s.next $t_{p} \neq x_{0}$. Thus when $p_{0}$ executes action check ${ }_{p}$ of Fig.2 for the first time after state $s_{1}$, the 
condition will be true, so in some subsequent transition $p_{0}$ will have to execute action $\mathrm{do}_{p_{0}, x_{0}}$, performing job $x_{0}$, which is a contradiction, since after state $s_{0}$ no jobs are executed.

Case A.2) After $s_{0}^{\prime \prime}$, process $p_{0}$ executes action compNext ${ }_{p_{0}}$ and the transition leads in state $s_{1}>s_{0}^{\prime \prime}$ such that $s_{1} \cdot \operatorname{NEXT}_{p_{0}}>x_{0}$. Since $p_{0}=\min _{p \in \mathcal{P}_{\alpha}}[p]$, it holds that $\forall x \in \mathrm{POOL}_{\alpha}$ such that $[x]_{\mathrm{POOL}_{\alpha}} \leq\left\lfloor\left(p_{0}-1\right) \cdot \frac{\left|\mathcal{J} \backslash \mathrm{DONE}_{\alpha}\right|-(m-1)}{m}\right\rfloor+1, \nexists p \in \mathcal{P}$ such that $s_{1} \cdot$ next $_{p}=x$. Let the transition $\left(s_{2}\right.$, compNext $\left._{p_{0}}, s_{2}^{\prime}\right) \in \alpha$, where $s_{2}>s_{1}$, be the first time that action compNext $p_{0}$ is executed after state $s_{1}$. We have that $\forall x \in$ $\operatorname{POOL}_{\alpha}$ such that $[x]_{\mathrm{POOL}_{\alpha}} \leq\left\lfloor\left(p_{0}-1\right) \cdot \frac{\left|\mathcal{J} \backslash \mathrm{DONE}_{\alpha}\right|-(m-1)}{m}\right\rfloor+1, x \notin s_{2} \cdot \mathrm{DONE}_{p_{0}} \cup$ $s_{2} \cdot \mathrm{TRY}_{p_{0}}$, since from the discussion above we have that $\forall s \geq s_{1}$ and $\forall p \in \mathcal{P}_{\alpha} \backslash\left\{p_{0}\right\}$, $\left[\text { s.next }_{p}\right]_{\mathrm{POOL}_{\alpha}} \geq\left\lfloor(p-1) \cdot \frac{\left|\mathcal{J} \backslash \mathrm{DONE}_{\alpha}\right|-(m-1)}{m}\right\rfloor+1$. Thus $\left[x_{0}\right]_{s_{2} . \mathrm{FREE}_{p_{0}} \backslash s_{2} . \mathrm{TRY}_{p_{0}}}=$ $\left[x_{0}\right]_{\mathrm{POOL}_{\alpha}}=\left\lfloor\left(p_{0}-1\right) \cdot \frac{\left|\mathcal{J} \backslash \mathrm{DONE}_{\alpha}\right|-(m-1)}{m}\right\rfloor+1$. As a result, $s_{2}^{\prime} \cdot \mathrm{NEXT}_{p_{0}}=x_{0}$. With similar arguments like in case A.1, we can see that job $x_{0}$ will be performed by process $p_{0}$, which is a contradiction, since after state $s_{0}$ no jobs are executed.

Case $\mathbf{B}\left|\mathcal{J} \backslash \mathrm{DONE}_{\alpha}\right|<2 m-1$ : Let $x_{0} \in \mathrm{POOL}_{\alpha}$ be the job such that $\left[x_{0}\right]_{\mathrm{POOL}_{\alpha}}=p_{0}$. Such $x_{0}$ exists since $\beta \geq m$ and $\mathrm{POOL}_{\alpha} \geq \beta$. It follows that any $p \in \mathcal{P}_{\alpha}$ that executes action compNext ${ }_{p}$ after state $s_{0}^{\prime}$, will have its $\operatorname{NEXT}_{p}$ variable pointing in a job $x$ with $[x]_{\mathrm{POOL}_{\alpha}} \geq p$. Thus $\forall p \in \mathcal{P}_{\alpha}, \exists s_{p}^{\prime} \geq s_{0}^{\prime}$ in $\alpha$ such that $\forall$ states $s \geq s_{p}^{\prime}$, $[\text { s.next }]_{\mathrm{POOL}_{\alpha}} \geq p$. Let $s_{0}^{\prime \prime}=$ $\max _{p \in \mathcal{P}_{\alpha}}\left[s_{p}^{\prime}\right]$, we have 2 cases for $p_{0}$ :

Case B.1) After $s_{0}^{\prime \prime}$, process $p_{0}$ executes action compNext $p_{0}$ and the transition leads in state $s_{1}>s_{0}^{\prime \prime}$ such that $s_{1} \cdot \mathrm{NEXT}_{p_{0}}=x_{0}$. Since $\left[x_{0}\right]_{\mathrm{POOL}_{\alpha}}=p_{0}$ and $p_{0}=\min _{p \in \mathcal{P}_{\alpha}}[p]$, from the previous discussion we have that $\forall s \geq s_{1}$ and $\forall p \in \mathcal{P} \backslash\left\{p_{0}\right\}$, s.next $t_{p} \neq x_{0}$. Thus when $p_{0}$ executes action check $k_{p}$ of Fig.2] for the first time after state $s_{1}$, the condition will be true, so in some subsequent transition $p_{0}$ will have to execute action $\mathrm{do}_{p_{0}, x_{0}}$, performing job $x_{0}$, which is a contradiction, since after state $s_{0}$ no jobs are executed.

Case B.2) After $s_{0}^{\prime \prime}$, process $p_{0}$ executes action compNext $p_{0}$ and the transition leads in state $s_{1}>s_{0}^{\prime \prime}$ such that $s_{1} \cdot \operatorname{NEXT}_{p_{0}}>x_{0}$. Since $p_{0}=\min _{p \in \mathcal{P}_{\alpha}}[p]$, it holds that $\forall x \in \mathrm{POOL}_{\alpha}$ such that $[x]_{\mathrm{POOL}_{\alpha}} \leq p_{0}, \nexists p \in \mathcal{P}$ such that $s_{1}$. next $_{p}=x$. Let the transition $\left(s_{2}\right.$, compNext $\left._{p_{0}}, s_{2}^{\prime}\right) \in$ $\alpha$, where $s_{2}>s_{1}$, be the first time that action compNext $p_{0}$ is executed after state $s_{1}$. We have that $\forall x \in \mathrm{POOL}_{\alpha}$ such that $[x]_{\mathrm{POOL}_{\alpha}} \leq p_{0}, x \notin s_{2}$. DONE $p_{0} \cup s_{2}$. TRY $p_{0}$, since from the discussion above we have that $\forall s \geq s_{1}$ and $\forall p \in \mathcal{P}_{\alpha} \backslash\left\{p_{0}\right\}$, [s.next $]_{\mathrm{POOL}_{\alpha}} \geq p$. Thus $\left[x_{0}\right]_{s_{2} \cdot \mathrm{FREE}_{p_{0}} \backslash s_{2} \cdot \mathrm{TRY}_{p_{0}}}=\left[x_{0}\right]_{\mathrm{POOL}_{\alpha}}=p_{0}$. As a result, $s_{2}^{\prime} \cdot \operatorname{NEXT}_{p_{0}}=x_{0}$. With similar arguments like in case B.1, we can see that job $x_{0}$ will be performed by process $p_{0}$, which is a contradiction, since after state $s_{0}$ no jobs are executed.

We combine the last two lemmas in order to show the main result on the effectiveness of algorithm $\mathrm{KK}_{\beta}$.

Theorem 4.4. For any $\beta \geq m, f \leq m-1$ algorithm $\mathrm{KK}_{\beta}$ has effectiveness $E_{\mathrm{KK}_{\beta}}(n, m, f)=$ $n-(\beta+m-2)$.

Proof. From Lemma 4.2 we have that any finite execution $\alpha \in$ execs $\left(\mathrm{KK}_{\beta}\right)$ with $D o(\alpha) \leq$ $n-(\beta+m-1)$ can be extended, essentially proving that in such executions no process has terminated. Moreover from Lemma 4.3 we have that $\mathrm{KK}_{\beta}$ is wait free, and thus there exists no infinite fair execution $\alpha \in \operatorname{execs}\left(\mathrm{KK}_{\beta}\right)$, such that $D o(\alpha) \leq n-(\beta+m-1)$. Since finite 
fair executions are executions where all non-failed processes have terminated, from the above we have that $E_{\mathrm{KK}_{\beta}}(n, m, f) \geq n-(\beta+m-2)$.

If all processes but the process with id $m$ fail in an execution $\alpha$ in such a way that $\mathcal{J}_{\alpha} \cap \mathrm{STUCK}_{\alpha}=\emptyset$ and $\left|\mathrm{STUCK}_{\alpha}\right|=m-1$ (where STUCK $_{\alpha}$ is defined as in the proof of Lemma 4.3), it is easy to see that there exists an adversarial strategy, such that when process $m$ terminates, $\beta+m-2$ jobs have not been performed. Such an execution will be a finite fair execution where $n-(\beta+m-2)$ jobs are performed. Thus we have that $E_{\mathrm{KK}_{\beta}}(n, m, f)=n-(\beta+m-2)$.

\section{Work Complexity Analysis}

In this section we are going to prove that for $\beta \geq 3 m^{2}$ algorithm $\mathrm{KK}_{\beta}$ has work complexity $\mathrm{O}(n m \log n \log m)$.

The main idea of the proof, is to demonstrate that under the assumption $\beta \geq 3 m^{2}$, process collisions on a job cannot accrue without making progress in the algorithm. In order to prove that, we first demonstrate in Lemma5.1 that if two different processes $p, q$ set their $\mathrm{NEXT}_{p}, \mathrm{NEXT}_{q}$ internal variables to the same job $i$ in some compNext actions, then the $\mathrm{DONE}_{p}$ and $\mathrm{DONE}_{q}$ sets of the processes, have at least $|q-p| \cdot m$ different elements, given that $\beta \geq 3 m^{2}$. Next we prove in Lemma 5.4 that if two processes $p, q$ collide three consecutive times, while trying to perform some jobs, the size of the set $\mathrm{DONE}_{p} \cup \mathrm{DONE}_{q}$ that processes $p$ and $q$ know will increase by at least $|q-p| \cdot m$ elements. This essentially tells us that every three collisions between the same two processes a significant number of jobs has been performed, and thus enough progress has been made. In order to prove the above statement, we formally define what we mean by collision in Definition 5.2. and tie such a collision with some specific state, the state the collision is detected, so that we have a fixed "point of reference" in the execution; and show that the order collisions are detected in an execution, is consistent with the order the involved processes attempt to perform the respective jobs in Lemmas 5.2, 5.3. Finally we use Lemma5.4 in order to prove in Lemma 5.5 , that a process $p$ cannot collide with a process $q$ more than $2\left[\frac{n}{m \cdot|q-p|}\right]$ times in any execution. This is proven by contradiction, showing that if process $p$ collides with process $q$ more than $2\left[\frac{n}{m \cdot|q-p|}\right]$ times, there exist states for which the set $\left|\mathrm{DONE}_{p} \cup \mathrm{DONE}_{q}\right|$ has more than $n$ elements which is impossible. Lemma5.5 is used in order to prove the main result on the work complexity of algorithm $\mathrm{KK}_{\beta}$ for $\beta \geq 3 m^{2}$, Theorem5.6 We obtain Theorem5.6 by counting the total number of collisions that can happen and the cost of each collision.

We start by defining the notion of immediate predecessor transition for a state $s$ in an execution $\alpha$. The immediate predecessor is the last transition of a specific action type that precedes state $s$ in the execution. This is particularly useful in uniquely identifying the transition with action compNext ${ }_{p}$ in an execution, that last set a $\operatorname{NEXT}_{p}$ internal variable to a specific value, given a state $s$ of interest.

Definition 5.1. We say that transition $\left(s_{1}, \pi_{1}, s_{1}^{\prime}\right)$ is an immediate predecessor of state $s_{2}$ in an execution $\alpha \in \operatorname{execs}\left(\mathrm{KK}_{\beta}\right)$ and we write $\left(s_{1}, \pi_{1}, s_{1}^{\prime}\right) \mapsto s_{2}$, if $s_{1}^{\prime}<s_{2}$ and in the execution fragment $\alpha^{\prime}$ that begins with state $s_{1}^{\prime}$ and ends with state $s_{2}$, there exists no action $\pi_{3}=\pi_{1}$.

Next we define what a collision between two processes means. We say that process $p$ collided with process $q$ in job $i$ at state $s$, if process $p$ attempted to preform job $i$, but was not able to, 
because it detected in state $s$ that either process $q$ was trying to perform job $i$ or process $q$ has already performed job $i$.

Definition 5.2. In an execution $\alpha \in \operatorname{execs}\left(\mathrm{KK}_{\beta}\right)$, we say that process $p$ collided with process $q$ in job $i$ at state $s$, if $(i)$ there exist in $\alpha$ transitions $\left(s_{1}\right.$, compNext $\left._{p}, s_{1}^{\prime}\right),\left(t_{1}, \operatorname{compNext}_{q}, t_{1}^{\prime}\right)$ and $\left(s_{2}, \operatorname{check}_{p}, s_{2}^{\prime}\right)$, where $\left(s_{1}, \operatorname{compNext}_{p}, s_{1}^{\prime}\right) \mapsto s_{2}, t_{1}<s_{2}$ and $s_{1}^{\prime} \cdot \mathrm{NEXT}_{p}=t_{1}^{\prime} \cdot \mathrm{NEXT}_{q}=$ $s_{2} \cdot \mathrm{NEXT}_{p}=i, s_{1}^{\prime} \cdot \mathrm{STATUS}_{p}=t_{1}^{\prime} \cdot \mathrm{STATUS}_{q}=$ set_next, $s_{2}^{\prime} \cdot$ STATUS $_{p}=$ comp_next, (ii) in execution fragment $\alpha^{\prime}=s_{1}^{\prime}, \ldots, s_{2}$ either there exists transition $\left(s\right.$, gatherTry $\left._{p}, s^{\prime}\right)$ such that $s . \mathrm{Q}_{p}=q$, s.next $q=i$, or transition $\left(s\right.$, gatherDone $\left._{p}, s^{\prime}\right)$ and $j \in\{1, \ldots, n\}$ such that $s . \mathrm{Q}_{p}=$ $q, s \cdot \operatorname{POS}_{p}(q)=j$, s.done $q, j=i$ and $i \notin s . \mathrm{TRY}_{p}$.

Definition 5.3. In an execution $\alpha \in \operatorname{execs}\left(\mathrm{KK}_{\beta}\right)$, we say that processes $p, q$ collide in job $i$ at state $s$, if process $p$ collided with process $q$ or process $q$ collided with process $p$ in job $i$ at state $s$, according to Definition 5.2

Next we show that if two processes $p, q$ decide, with some compNext actions, to perform the same job $i$, then their DONE sets at the enabling states of those compNext actions, differ in at-least $|q-p| \cdot m$ elements.

Lemma 5.1. If $\beta \geq 3 m^{2}$ and in an execution $\alpha \in \operatorname{execs}\left(\mathrm{KK}_{\beta}\right)$ there exist states $s_{1}, t_{1}$ and processes $p, q \in \mathcal{P}$ with $p<q$ such that $s_{1} \cdot \mathrm{NEXT}_{p}=t_{1} \cdot \mathrm{NEXT}_{q}=i \in \mathcal{J}$, then there exist transitions $\left(s_{2}\right.$, compNext $\left._{p}, s_{2}^{\prime}\right) \mapsto s_{1},\left(t_{2}\right.$, compNext $\left._{q}, t_{2}^{\prime}\right) \mapsto t_{1}$, where $s_{2}^{\prime} \cdot \mathrm{NEXT}_{p}=t_{2}^{\prime} \cdot \mathrm{NEXT}_{q}=i$, $s_{2}^{\prime} \cdot$ STATUS $_{p}=t_{2}^{\prime} \cdot$ STATUS $_{q}=$ set_next and $\left|s_{2} \cdot \mathrm{DONE}_{p} \cap \overline{t_{2} \cdot \mathrm{DONE}_{q}}\right|>(q-p) \cdot m$ or $\left|\overline{s_{2} \cdot \mathrm{DONE}_{p}} \cap t_{2} \cdot \mathrm{DONE}_{q}\right|>(q-p) \cdot m$.

Proof. We will prove this by contradiction. From algorithm $\mathrm{KK}_{\beta}$ there must exist transitions $\left(s_{2}\right.$, compNext $\left._{p}, s_{2}^{\prime}\right) \mapsto s_{1}$ and $\left(t_{2}\right.$, compNext $\left._{q}, t_{2}^{\prime}\right) \mapsto t_{1}$, where $s_{2}^{\prime} \cdot \operatorname{NEXT}_{p}=i$ and $t_{2}^{\prime} \cdot \operatorname{NEXT}_{q}=i$, if there exist $s_{1}, t_{1} \in \alpha$ and $p, q \in \mathcal{P}$ with $p<q$ such that $s_{1} \cdot \mathrm{NEXT}_{p}=$ $t_{1} \cdot \operatorname{NEXT}_{q}=i \in \mathcal{J}$, since those are the transitions that set $\operatorname{NEXT}_{p}$ and $\operatorname{NEXT}_{q}$ to $i$. In order to get a contradiction we assume that $\left|s_{2} . \mathrm{DONE}_{p} \cap \overline{t_{2} \cdot \mathrm{DONE}_{q}}\right| \leq(q-p) \cdot m$ and $\left|\overline{s_{2} \cdot \mathrm{DONE}_{p}} \cap t_{2} \cdot \mathrm{DONE}_{q}\right| \leq(q-p) \cdot m$. We will prove that if this is the case, then $s_{2}^{\prime} \cdot \mathrm{NEXT}_{p} \neq t_{2}^{\prime} \cdot \mathrm{NEXT}_{q}$.

Let $\mathrm{A}=\mathcal{J} \backslash s_{2} \cdot \mathrm{DONE}_{p}=s_{2} \cdot \mathrm{FREE}_{p}$ and $\mathrm{B}=\mathcal{J} \backslash t_{2} \cdot \mathrm{DONE}_{q}=t_{2} \cdot \mathrm{FREE}_{q}$, thus from the contradiction assumption we have that: $|\overline{\mathrm{A}} \cap \mathrm{B}| \leq(q-p) \cdot m$ and $|\mathrm{A} \cap \overline{\mathrm{B}}| \leq(q-p) \cdot m$.

It could either be that $|\mathrm{A}|<|\mathrm{B}|$ or $|\mathrm{A}| \geq|\mathrm{B}|$.

Case $1|\mathrm{~A}|<|\mathrm{B}|$ : From the contradiction assumption we have that $|\overline{\mathrm{A}} \cap \mathrm{B}| \leq(q-p) \cdot m$. Since $s_{2} . \mathrm{FREE}_{p} \backslash s_{2} \cdot \mathrm{TRY}_{p}$ can have up to $m-1$ fewer elements than $\mathrm{A}-$ the elements of set $s_{2} \cdot \mathrm{TRY}_{p}$ and it can be the case that $s_{2} \cdot \mathrm{TRY}_{p} \cap t_{2} \cdot \mathrm{TRY}_{q}=\emptyset$, we have:

$$
\left|t_{2} \cdot \mathrm{FREE}_{q} \backslash t_{2} \cdot \mathrm{TRY}_{q} \cap \overline{s_{2} \cdot \mathrm{FREE}_{p} \backslash s_{2} \cdot \mathrm{TRY}_{p}}\right| \leq m(q-p)+m-1
$$

Moreover, since $s_{2} \cdot \mathrm{FREE}_{p} \backslash s_{2} \cdot \mathrm{TRY}_{p} \subseteq \mathrm{A}$ and $\left|s_{2} \cdot \mathrm{FREE}_{p} \backslash s_{2} \cdot \mathrm{TRY}_{p}\right| \geq \beta \geq 3 m^{2},|\mathrm{~A}| \geq$ $3 m^{2}$. Similarly $|\mathrm{B}| \geq 3 m^{2}$. We have:

$$
(q-1) \frac{|\mathrm{B}|}{m}=(p-1) \frac{|\mathrm{B}|}{m}+(q-p) \frac{|\mathrm{B}|}{m}>(p-1) \frac{|\mathrm{A}|}{m}+(q-p) \frac{|\mathrm{B}|}{m} \Rightarrow
$$




$$
\begin{gathered}
\Rightarrow(q-1) \frac{|\mathrm{B}|}{m}>(p-1) \frac{|\mathrm{A}|}{m}+3 m(q-p) \Rightarrow \\
\Rightarrow(q-1) \frac{|\mathrm{B}|}{m}>(p-1) \frac{|\mathrm{A}|}{m}+(3 m-1)(q-p)+(q-p) \Rightarrow \\
\Rightarrow(q-1) \frac{|\mathrm{B}|}{m}>(p-1) \frac{|\mathrm{A}|}{m}+(3 m-1)(q-p)+\frac{(q-p)(m-1)}{m} \Rightarrow \\
\left\lfloor(q-1) \frac{|\mathrm{B}|-(m-1)}{m}\right\rfloor+1 \geq\left\lfloor(p-1) \frac{|\mathrm{A}|-(m-1)}{m}\right\rfloor+1+(3 m-1)(q-p)
\end{gathered}
$$

Since $s_{2}^{\prime} \cdot \operatorname{NEXT}_{p}=t_{2}^{\prime} \cdot \operatorname{NEXT}_{q}=i$, we have:

$$
\begin{aligned}
& {[i]_{s_{2} \cdot \mathrm{FREE}_{p} \backslash s_{2} \cdot \mathrm{TRY}_{p}}=\left\lfloor(p-1) \frac{|\mathrm{A}|-(m-1)}{m}\right\rfloor+1} \\
& {[i]_{t_{2} . \mathrm{FREE}_{q} \backslash t_{2} \cdot \mathrm{TRY}_{q}}=\left\lfloor(q-1) \frac{|\mathrm{B}|-(m-1)}{m}\right\rfloor+1}
\end{aligned}
$$

Equation2 2 becomes:

$$
[i]_{t_{2} \cdot \operatorname{FREE}_{q} \backslash t_{2} \cdot \mathrm{TRY}_{q}} \geq[i]_{s_{2} \cdot \mathrm{FREE}_{p} \backslash s_{2} \cdot \mathrm{TRY}_{p}}+(3 m-1)(q-p)
$$

Thus set $t_{2} \cdot \mathrm{FREE}_{q} \backslash t_{2} \cdot \mathrm{TRY}_{q}$ must have at least $(3 m-1)(q-p)$ more elements with rank less that the rank of $i$, than set $s_{2} \cdot \mathrm{FREE}_{p} \backslash s_{2} \cdot \mathrm{TRY}_{p}$ does. This is a contradiction since from eq.1 we have that:

$$
\left|t_{2} \cdot \mathrm{FREE}_{q} \backslash t_{2} \cdot \mathrm{TRY}_{q} \cap \overline{s_{2} \cdot \mathrm{FREE}_{p} \backslash s_{2} \cdot \mathrm{TRY}_{p}}\right| \leq m(q-p)+m-1
$$

Case $2|\mathrm{~B}| \leq|\mathrm{A}|$ : We have that $|\overline{\mathrm{A}} \cap \mathrm{B}| \leq(q-p) \cdot m$ and $|\mathrm{A} \cap \overline{\mathrm{B}}| \leq(q-p) \cdot m$ from the contradiction assumption. Since $s_{2} . \mathrm{FREE}_{p} \backslash s_{2}$. TRY $p$ can have up to $m-1$ less elements than A - the elements of set $s_{2} \cdot \mathrm{TRY}_{p}$ - and it can be the case that $s_{2} \cdot \operatorname{TRY}_{p} \cap t_{2} \cdot \operatorname{TRY}_{p}=\emptyset$, we have:

$$
\left|t_{2} \cdot \mathrm{FREE}_{q} \backslash t_{2} \cdot \mathrm{TRY}_{q} \cap \overline{s_{2} \cdot \mathrm{FREE}_{p} \backslash s_{2} \cdot \mathrm{TRY}_{p}}\right| \leq m(q-p)+m-1
$$

From the contradiction assumption and the case 2 assumption we have that $|\mathrm{B}| \leq|\mathrm{A}| \leq|\mathrm{B}|+$ $(q-p) \cdot m$. Moreover $|\mathrm{A}| \geq \beta \geq 3 m^{2}$ and $|\mathrm{B}| \geq \beta \geq 3 m^{2}$. We have:

$$
\begin{gathered}
(q-1) \frac{|\mathrm{B}|+(q-p) m}{m}=(p-1) \frac{|\mathrm{B}|+(q-p) m}{m}+(q-p) \frac{|\mathrm{B}|+(q-p) m}{m} \geq \\
\geq(p-1) \frac{|\mathrm{A}|}{m}+(q-p) \frac{|\mathrm{B}|+(q-p) m}{m} \geq(p-1) \frac{|\mathrm{A}|}{m}+3 m(q-p)+(q-p)^{2} \Rightarrow \\
\Rightarrow(q-1) \frac{|\mathrm{B}|}{m} \geq(p-1) \frac{|\mathrm{A}|}{m}+3 m(q-p)+(q-p)^{2}-(q-1)(q-p) \Rightarrow \\
\Rightarrow(q-1) \frac{|\mathrm{B}|}{m} \geq(p-1) \frac{|\mathrm{A}|}{m}+(3 m-p+1)(q-p) \Rightarrow \\
\Rightarrow(q-1) \frac{|\mathrm{B}|}{m} \geq(p-1) \frac{|\mathrm{A}|}{m}+(2 m+2)(q-p) \Rightarrow
\end{gathered}
$$




$$
\begin{gathered}
\Rightarrow(q-1) \frac{|\mathrm{B}|}{m} \geq(p-1) \frac{|\mathrm{A}|}{m}+(2 m+1)(q-p)+\frac{(q-p)(m-1)}{m} \Rightarrow \\
\left\lfloor(q-1) \frac{|\mathrm{B}|-(m-1)}{m}\right\rfloor+1 \geq\left\lfloor(p-1) \frac{|\mathrm{A}|-(m-1)}{m}\right\rfloor+1+(2 m+1)(q-p)
\end{gathered}
$$

Since $s_{2}^{\prime} \cdot \operatorname{NEXT}_{p}=t_{2}^{\prime} \cdot \operatorname{NEXT}_{q}=i$, we have:

$$
\begin{aligned}
& {[i]_{s_{2} . \mathrm{FREE}_{p} \backslash s_{2} \cdot \mathrm{TRY}_{p}}=\left\lfloor(p-1) \frac{|\mathrm{A}|-(m-1)}{m}\right\rfloor+1} \\
& {[i]_{t_{2} . \mathrm{FREE}_{q} \backslash t_{2} . \mathrm{TRY}_{q}}=\left\lfloor(q-1) \frac{|\mathrm{B}|-(m-1)}{m}\right\rfloor+1}
\end{aligned}
$$

Equation 4 becomes:

$$
[i]_{t_{2} . \mathrm{FREE}_{q} \backslash t_{2} . \mathrm{TRY}_{q}} \geq[i]_{s_{2} . \mathrm{FREE}_{p} \backslash s_{2} \cdot \mathrm{TRY}_{p}}+(2 m+1)(q-p)
$$

Thus set $t_{2} \cdot \mathrm{FREE}_{q} \backslash t_{2} \cdot \mathrm{TRY}_{q}$ must have at least $(2 m+1)(q-p)$ more elements with rank less that the rank of $i$, than set $s_{2} \cdot \mathrm{FREE}_{p} \backslash s_{2} \cdot \mathrm{TRY}_{p}$. This is a contradiction since from eq.3 we have that:

$$
\left|t_{2} \cdot \mathrm{FREE}_{q} \backslash t_{2} \cdot \mathrm{TRY}_{q} \cap \overline{s_{2} \cdot \mathrm{FREE}_{p} \backslash s_{2} \cdot \mathrm{TRY}_{p}}\right| \leq m(q-p)+m-1
$$

Next we show that if a process $p$ detects consecutive collisions with process $q$, the processes $p, q$ attempted to perform the jobs associated with the collisions in the same order and the order process $p$ detects the collisions according to Definition5.2 is the same as the order processes $p, q$ attempted to perform the jobs.

In the proofs that follow, for a state $s$ in execution $\alpha$ we define as $s$.DONE the following set: $s . \mathrm{DONE}=\left\{i \in \mathcal{J} \mid \exists p \in \mathcal{P}\right.$ and $\left.j \in\{1, \ldots, n\}: s . \operatorname{done}_{p}(j)=i\right\}$.

Lemma 5.2. In an execution $\alpha \in \operatorname{execs}\left(\mathrm{KK}_{\beta}\right)$ for any $\beta \geq m$ if there exist processes $p, q$, jobs $i_{1}, i_{2} \in \mathcal{J}$ and states $\tilde{s}_{1}<\tilde{s}_{2}$ such that process $p$ collided with process $q$ in job $i_{1}$ at state $\tilde{s}_{1}$ and in job $i_{2}$ at state $\tilde{s}_{2}$ according to Definition 5.2 then there exist transitions $\left(s_{1}, \operatorname{compNext}_{p}, s_{1}^{\prime}\right) \mapsto \tilde{s}_{1},\left(s_{2}, \operatorname{compNext}_{p}, s_{2}^{\prime}\right) \mapsto \tilde{s}_{2}$ and $\left(t_{1}, \operatorname{compNext}_{q}, t_{1}^{\prime}\right)$, $\left(t_{2}\right.$, compNext $\left._{q}, t_{2}^{\prime}\right)$ where $s_{1}^{\prime} \cdot \mathrm{NEXT}_{p}=t_{1}^{\prime} \cdot \mathrm{NEXT}_{q}=i_{1}, s_{2}^{\prime} \cdot \mathrm{NEXT}_{p}=t_{2}^{\prime} \cdot \mathrm{NEXT}_{q}=i_{2}$, $s_{1}^{\prime} \cdot \operatorname{STATUS}_{p}=s_{2}^{\prime} \cdot \operatorname{STATUS}_{p}=t_{1}^{\prime} \cdot \operatorname{STATUS}_{q}=t_{2}^{\prime} \cdot \operatorname{STATUS}_{q}=$ set_next such that:

$$
s_{1}<s_{2} \text { and } t_{1}<t_{2} \text {. }
$$

Proof. From Definition 5.2 we have that there exist transitions $\left(s_{1}\right.$, compNext $\left._{p}, s_{1}^{\prime}\right)$, $\left(s_{2}, \operatorname{compNext}_{p}, s_{2}^{\prime}\right)$ with $s_{1}^{\prime} \cdot \operatorname{NEXT}_{p}=i_{1}, s_{2}^{\prime} \cdot \operatorname{NEXT}_{p}=i_{2}, s_{1}^{\prime} \cdot \operatorname{STATUS}_{p}=s_{2}^{\prime} \cdot \operatorname{STATUS}_{p}=$ set_next, and there exists no action $\pi_{1}=$ compNext $_{p}$ for which $s_{1}<\pi_{1}<\tilde{s}_{1}$ or $s_{2}<\pi_{1}<\tilde{s}_{2}$. From the latter and the fact that $\tilde{s}_{1}<\tilde{s}_{2}$, it must be the case that $s_{1}<\tilde{s}_{1}<s_{2}<\tilde{s}_{2}$. Furthermore from Definition 5.2 we have that there exist transitions $\left(t_{1}\right.$, compNext $\left._{q}, t_{1}^{\prime}\right)$, $\left(t_{2}, \operatorname{compNext}_{q}, t_{2}^{\prime}\right)$ with $t_{1}^{\prime} \cdot \mathrm{NEXT}_{q}=i_{1}, t_{2}^{\prime} \cdot \mathrm{NEXT}_{q}=i_{2}, t_{1}^{\prime} \cdot \operatorname{STATUS}_{q}=t_{2}^{\prime} \cdot \operatorname{STATUS}_{q}=$ 
set_next, such that $t_{1}^{\prime}<\tilde{s}_{1}$ and $t_{2}^{\prime}<\tilde{s}_{2}$. We can pick those transitions in $\alpha$ in such a way that there exists no other transition between $t_{1}^{\prime}$ and $\tilde{s}_{1}$ that sets $\operatorname{NEXT}_{q}$ to $i_{1}$ and similarly there exists no other transition between $t_{2}^{\prime}$ and $\tilde{s}_{2}$ that sets $\operatorname{NEXT}_{q}$ to $i_{2}$. We need to prove now that $t_{1}<t_{2}$. We will prove this by contradiction.

Let $t_{2}<t_{1}$. Since $t_{1}^{\prime}<\tilde{s}_{1}$, we have that $t_{2}<t_{1}<t_{1}^{\prime}<\tilde{s}_{1}<s_{2}<\tilde{s}_{2}$. Since from Definition[5.2 either $\tilde{s}_{1} . n e x t_{q}=i_{1}$ or there exists $j \in\{1, \ldots, n\}$ such that $\tilde{s}_{1}$. done $_{q, j}=i_{1}$, it must be the case that $\tilde{s}_{2} \cdot$ STATUS $_{p}=$ gather_done, $\tilde{s}_{2} \cdot \mathrm{Q}_{p}=q$ and there exists $j^{\prime} \in\{1, \ldots, n\}$ such that $\tilde{s}_{2}$. done $_{q, j^{\prime}}=i_{2}$. Essentially, it must be that case that process $q$ performed job $i_{2}$ after transition $\left(t_{2}\right.$, compNext $\left._{q}, t_{2}^{\prime}\right)$. This means that there exists transition $\left(t_{3}\right.$, done $\left._{q}, t_{3}^{\prime}\right)$ and $j^{\prime} \in\{1, \ldots, n\}$ such that $t_{3}^{\prime}$. done $e_{q, j^{\prime}}=i_{2}$ and $t_{2}<t_{3}^{\prime}<t_{1}<t_{1}^{\prime}<\tilde{s}_{1}<s_{2}<\tilde{s}_{2}$.

If $\tilde{s}_{1}$. STATUS $_{p}=$ gather_try then from algorithm $\mathrm{KK}_{\beta}$ we have that $\tilde{s}_{1}$.DONE $\subseteq$ $s_{2}$. $\mathrm{DONE}_{p}$, since actions gatherTry $p_{p}$ are followed by actions gatherDone $e_{p}$ before any action setNext $p_{p}$ takes place. As a result $i_{2} \in s_{2} \cdot \mathrm{DONE}_{p}$, which is a contradiction since $\left(s_{2}, \operatorname{compNext}_{p}, s_{2}^{\prime}\right) \notin \operatorname{trans}\left(\mathrm{KK}_{\beta}\right)$ if $i_{2} \in s_{2} \cdot \mathrm{DONE}_{p}$ and $s_{2}^{\prime} \cdot \mathrm{NEXT}_{p}=i_{2}, s_{2}^{\prime} \cdot \operatorname{STATUS}_{p}=$ set_next.

If $\tilde{s}_{1}$.STATUS ${ }_{p}=$ gather_done then from algorithm $\mathrm{KK}_{\beta}$ we have that $\tilde{s}_{1} \cdot \mathrm{Q}_{p}=q$ and there exists $j \in\{1, \ldots, n\}$ such that $\tilde{s}_{1} \cdot \operatorname{POS}_{p}(q)=j$ and $\tilde{s}_{1}$.done $e_{q, j}=i_{1}$. Since $t_{2}<t_{3}^{\prime}<t_{1}<$ $t_{1}^{\prime}<\tilde{s}_{1}<s_{2}<\tilde{s}_{2}$ it must be the case that $j^{\prime}<j$ and as a result $i_{2} \in \tilde{s}_{1} \cdot \operatorname{DONE}_{p}$. Clearly $\tilde{s}_{1} \cdot \mathrm{DONE}_{p} \subseteq s_{2}$. $\mathrm{DONE}_{p}$, which is a contradiction since $\left(s_{2}, \operatorname{compNext}_{p}, s_{2}^{\prime}\right) \notin \operatorname{trans}\left(\mathrm{KK}_{\beta}\right)$ if $i_{2} \in s_{2}$.DONE ${ }_{p}$ and $s_{2}^{\prime} \cdot \operatorname{NEXT}_{p}=i_{2}, s_{2}^{\prime}$. STATUS $_{p}=$ set_next.

Next we show that if two consecutive collisions take place between processes $p, q$, and $p$ detects the one collision and $q$ the other, the processes $p, q$ attempted to perform the jobs associated with the collisions in the same order and the order in which the processes detect the collisions according to Definition 5.2 is the same as the order the processes $p, q$ attempted to perform the jobs.

Lemma 5.3. In an execution $\alpha \in \operatorname{execs}\left(\mathrm{KK}_{\beta}\right)$ for any $\beta \geq m$ if there exist processes $p$, $q$, jobs $i_{1}, i_{2} \in \mathcal{J}$ and states $\tilde{s}_{1}<\tilde{s}_{2}$ such that process $p$ collided with process $q$ in job $i_{1}$ at state $\tilde{s}_{1}$ and process $q$ collided with process $p$ in job $i_{2}$ at state $\tilde{s}_{2}$ according to Definition[5.2 then there exist transitions $\left(s_{1}\right.$, compNext $\left._{p}, s_{1}^{\prime}\right) \mapsto \tilde{s}_{1},\left(t_{2}\right.$, compNext $\left._{q}, t_{2}^{\prime}\right) \mapsto \tilde{s}_{2}$ and $\left(s_{2}, \operatorname{compNext}_{p}, s_{2}^{\prime}\right)$, $\left(t_{1}\right.$, compNext $\left._{q}, t_{1}^{\prime}\right)$, where $s_{1}^{\prime} \cdot \mathrm{NEXT}_{p}=t_{1}^{\prime} \cdot \mathrm{NEXT}_{q}=i_{1}, s_{2}^{\prime} \cdot \mathrm{NEXT}_{p}=t_{2}^{\prime} \cdot \mathrm{NEXT}_{q}=i_{2}$, $s_{1}^{\prime} \cdot$ STATUS $_{p}=s_{2}^{\prime} \cdot$ STATUS $_{p}=t_{1}^{\prime} \cdot$ STATUS $_{q}=t_{2}^{\prime} \cdot$ STATUS $_{q}=$ set_next such that:

$$
s_{1}<s_{2} \text { and } t_{1}<t_{2} .
$$

Proof. From Definition 5.2 we have that there exist transitions $\left(s_{1}\right.$, compNext $\left._{p}, s_{1}^{\prime}\right)$, $\left(s_{2}, \operatorname{compNext}_{p}, s_{2}^{\prime}\right)$ with $s_{1}^{\prime} \cdot \operatorname{NEXT}_{p}=i_{1}, s_{2}^{\prime} \cdot \operatorname{NEXT}_{p}=i_{2}, s_{1}^{\prime} \cdot \operatorname{STATUS}_{p}=s_{2}^{\prime} \cdot \operatorname{STATUS}_{p}=$ set_next, and there exists no action $\pi_{1}=\operatorname{compNext}_{p}$ for which $s_{1}<\pi_{1}<\tilde{s}_{1}$. Furthermore from Definition 5.2 we have that there exist transitions $\left(t_{1}\right.$, compNext $\left._{q}, t_{1}^{\prime}\right)$, $\left(t_{2}, \operatorname{compNext}_{q}, t_{2}^{\prime}\right)$ with $t_{1}^{\prime} \cdot \mathrm{NEXT}_{q}=i_{1}, t_{2}^{\prime} \cdot \mathrm{NEXT}_{q}=i_{2}, t_{1}^{\prime} \cdot \operatorname{STATUS}_{q}=t_{2}^{\prime} \cdot \operatorname{STATUS}_{q}=$ set_next, and there exists no action $\pi_{2}=\operatorname{compNext}_{q}$ for which $t_{2}<\pi_{2}<\tilde{s}_{2}$. From the later and the fact that $\tilde{s}_{1}<\tilde{s}_{2}$, it must be the case that $t_{1}<t_{2}<\tilde{s}_{2}$. We can pick the transitions 
that are enabled by states $t_{1}$ and $s_{2}$ in $\alpha$ in such a way that there exists no other transition between $t_{1}^{\prime}$ and $\tilde{s}_{1}$ that sets $\operatorname{NEXT}_{q}$ to $i_{1}$ and similarly there exists no other transition between $s_{2}^{\prime}$ and $\tilde{s}_{2}$ that sets $\operatorname{NEXT}_{p}$ to $i_{2}$. We need to prove now that $s_{1}<s_{2}$. We will prove this by contradiction.

Let $s_{2}<s_{1}$. From algorithm $\mathrm{KK}_{\beta}$ and Definition 5.2 there exist transitions $\left(s_{3}\right.$, setNext $\left._{p}, s_{3}^{\prime}\right)$, and $\left(t_{3}\right.$, setNext $\left._{q}, t_{3}^{\prime}\right)$, where $s_{3}^{\prime}$.next $t_{p}=i_{2}, t_{3}^{\prime}$.next $t_{q}=i_{1}$ and $s_{2}<$ $s_{3}^{\prime}<s_{1}, t_{1}<t_{3}^{\prime}<t_{2}$. There are 2 cases, either $s_{3}^{\prime}<t_{3}^{\prime}$ or $t_{3}^{\prime}<s_{3}^{\prime}$.

Case $1 s_{3}^{\prime}<t_{3}^{\prime}$ : We have that $s_{3}^{\prime}<t_{3}^{\prime}<t_{2}$ and $\left(t_{2}, \operatorname{compNext}_{q}, t_{2}^{\prime}\right)$, where $t_{2}^{\prime} \cdot \mathrm{NEXT}_{q}=i_{2}$ and $t_{2}^{\prime}$. $\operatorname{STATUS}_{q}=$ set_next which means that $i_{2} \notin t_{2} . \mathrm{TRY}_{q} \cup t_{2} . \mathrm{DONE}_{q}$. This is a contradiction since the $t_{2} . \mathrm{TRY}_{q}$ and $t_{2} . \mathrm{DONE}_{q}$ are computed by actions gatherTry ${ }_{q}$ and gatherDone ${ }_{q}$ that are preceded by state $s_{3}^{\prime}$. Either $i_{2} \in t_{2} \cdot \mathrm{TRY}_{q}$ or a new action setNext $t_{p}$ took place before the gatherTry $_{q}$ actions. In the latter case, if there is a transition $\left(s_{4}\right.$, done $\left._{p}, s_{4}^{\prime}\right)$, where $s_{4} \cdot n e x t_{p}=$ $i_{2}$, before the action setNext $t_{p}$, it must be the case that $i_{2} \in t_{2}$. DONE $q$. If there exists no such transition we have again a contradiction since we cannot have a collision in job $i_{2}$ at state $\tilde{s}_{2}$ as defined in Definition 5.2

Case $2 t_{3}^{\prime}<s_{3}^{\prime}$ : We have that $t_{3}^{\prime}<s_{3}^{\prime}<s_{1}$ and $\left(s_{1}\right.$, compNext $\left._{p}, s_{1}^{\prime}\right)$, where $s_{1}^{\prime} \cdot \mathrm{NEXT}_{p}=i_{1}$ and $s_{1}^{\prime}$.STATUS $S_{p}=$ set_next which means that $i_{1} \notin s_{1} \cdot \mathrm{TRY}_{p} \cup s_{1} \cdot \mathrm{DONE}_{p}$. This is a contradiction since the $s_{1} \cdot \mathrm{TRY}_{p}$ and $s_{1} \cdot \mathrm{DONE}_{p}$ sets are computed by gatherTry ${ }_{p}$ and gatherDone ${ }_{p}$ actions that are preceded by state $t_{3}^{\prime}$. Either $i_{1} \in s_{1} \cdot \mathrm{TRY}_{p}$ or a new action setNext took place before the gatherTry $\operatorname{Tations}_{p}$. In the latter case, if there is a transition $\left(t_{4}\right.$, done $\left._{q}, t_{4}^{\prime}\right)$, where $t_{4}$.next $t_{q}=i_{1}$, before the action setNext ${ }_{q}$, it must be the case that $i_{1} \in s_{1}$. DONE $p$. If there exists no such transition we have again a contradiction since we cannot have a collision in job $i_{1}$ at state $\tilde{s}_{1}$ as defined in Definition 5.2

Next we show that if 2 processes $p, q \in \mathcal{P}$ collide three times, their DONE sets at the third collision will contain at least $m \cdot(q-p)$ more jobs than they did at the first collision. This will allow us to find an upper bound on the collisions a process may participate in. It is possible that both processes become aware of a collision or only one of them does while the other one successfully completes the job.

Lemma 5.4. If $\beta \geq 3 m^{2}$ and in an execution $\alpha \in \operatorname{execs}\left(\mathrm{KK}_{\beta}\right)$ there exist processes $p \neq q$, jobs $i_{1}, i_{2}, i_{3} \in \mathcal{J}$ and states $\tilde{s}_{1}<\tilde{s}_{2}<\tilde{s}_{3}$ such that process $p, q$ collide in job $i_{1}$ at state $\tilde{s}_{1}$, in job $i_{2}$ at state $\tilde{s}_{2}$ and in job $i_{3}$ at state $\tilde{s}_{3}$ according to Definition 5.3 then there exist states $s_{1}<s_{3}$ and $t_{1}<t_{3}$ such that:

$$
\begin{gathered}
s_{1} \cdot \mathrm{DONE}_{p} \cup t_{1} \cdot \mathrm{DONE}_{q} \subseteq s_{3} \cdot \mathrm{DONE}_{p} \cap t_{3} \cdot \mathrm{DONE}_{q} \\
\left|s_{3} \cdot \mathrm{DONE}_{p} \cup t_{3} \cdot \mathrm{DONE}_{q}\right|-\left|s_{1} \cdot \mathrm{DONE}_{p} \cup t_{1} \cdot \mathrm{DONE}_{q}\right| \geq m \cdot|q-p|
\end{gathered}
$$

Proof. From Definitions 5.2 [5.3 we have that there exist transitions $\left(s_{1}\right.$, compNext $\left._{p}, s_{1}^{\prime}\right)$, $\left(s_{2}\right.$, compNext $\left._{p}, s_{2}^{\prime}\right),\left(s_{3}\right.$, compNext $\left._{p}, s_{3}^{\prime}\right)$ and $\left(t_{1}\right.$, compNext $\left._{q}, t_{1}^{\prime}\right),\left(t_{2}\right.$, compNext $\left._{q}, t_{2}^{\prime}\right)$, $\left(t_{3}, \operatorname{compNext}_{q}, t_{3}^{\prime}\right)$, where $s_{1}^{\prime} \cdot \mathrm{NEXT}_{p}=t_{1}^{\prime} \cdot \mathrm{NEXT}_{q}=i_{1}, s_{2}^{\prime} \cdot \mathrm{NEXT}_{p}=t_{2}^{\prime} \cdot \mathrm{NEXT}_{q}=i_{2}$, $s_{3}^{\prime} \cdot \operatorname{NEXT}_{p}=t_{3}^{\prime} \cdot \operatorname{NEXT}_{q}=i_{3}, s_{1}^{\prime} \cdot \operatorname{STATUS}_{p}=s_{2}^{\prime} \cdot \operatorname{STATUS}_{p}=s_{3}^{\prime} \cdot \operatorname{STATUS}_{p}=t_{1}^{\prime} \cdot$ STATUS $_{q}=$ $t_{2}^{\prime} \cdot \operatorname{STATUS}_{q}=t_{3}^{\prime} \cdot \operatorname{STATUS}_{q}=$ set_next and $s_{1}<\tilde{s}_{1}, t_{1}<\tilde{s}_{1}, s_{2}<\tilde{s}_{2}, t_{2}<\tilde{s}_{2}$, and $s_{3}<\tilde{s}_{3}$, 
$t_{3}<\tilde{s}_{3}$. We pick from $\alpha$ the transitions $\left(s_{1}, \operatorname{compNext}_{p}, s_{1}^{\prime}\right),\left(t_{1}, \operatorname{compNext}_{q}, t_{1}^{\prime}\right)$, in such a way that there exists no other compNext ${ }_{p}$, compNext ${ }_{q}$ between states $s_{1}, \tilde{s}_{1}$ respectively $t_{1}$, $\tilde{s}_{1}$ that sets $\operatorname{NEXT}_{p}$ respectively $\operatorname{NEXT}_{q}$ to $i_{1}$. We can pick in a similar manner the transitions for jobs $i_{2}, i_{3}$. From Lemmas 5.2, 5.3 and Definitions 5.2, 5.3 we have that $s_{1}<s_{2}<s_{3}$ and $t_{1}<t_{2}<t_{3}$. We will first prove that:

$$
s_{1} . \mathrm{DONE}_{p} \cup t_{1} \cdot \mathrm{DONE}_{q} \subseteq s_{3} . \mathrm{DONE}_{p} \cap t_{3} \cdot \mathrm{DONE}_{q}
$$

From algorithm $\mathrm{KK}_{\beta}$ we have that there exists in $\alpha$ transitions $\left(s_{4}, \operatorname{setNext}_{p}, s_{4}^{\prime}\right)$, $\left(t_{4}\right.$, setNext $\left._{q}, t_{4}^{\prime}\right)$ with $s_{4}^{\prime} . n e x t_{p}=i_{2}, t_{4}^{\prime} \cdot n e x t_{q}=i_{2}$ and there exist no action $\pi_{1}=$ compNext $t_{p}$, such that $s_{2}^{\prime}<\pi_{1}<s_{4}$, and no action $\pi_{2}=\operatorname{compNext}_{q}$, such that $t_{2}^{\prime}<\pi_{2}<t_{4}$. We need to prove that $t_{1}<s_{4}$ and $s_{1}<t_{4}$.

We start by proving that $t_{1}<s_{4}$. In order to get a contradiction we assume that $s_{4}<$ $t_{1}$. From algorithm $\mathrm{KK}_{\beta}$ we have that there exists in $\alpha$ transition $\left(t_{5}\right.$, gatherTry $\left.{ }_{q}, t_{5}^{\prime}\right)$, with $t_{5} \cdot \mathrm{Q}_{q}=p$, and there exists no action $\pi_{2}=\operatorname{compNext}_{q}$, such that $t_{5}^{\prime}<\pi_{2}<t_{2}$. We have that $s_{4}<t_{1}<t_{5}^{\prime}<t_{2}$ and $i_{2} \notin t_{2} . \mathrm{TRY}_{q} \cup t_{2}$. DONE $q$. If $t_{5}$.next $t_{p}=i_{2}$ we have a contradiction since $i_{2} \in s_{2}$. TRY ${ }_{q}$. If $t_{5}$.next $t_{q} \neq i_{2}$ there exists an action $\pi_{3}=\operatorname{setNext}_{p}$ in $\alpha$, such that $s_{4}<\pi_{3}<t_{5}$. If this $\pi_{3}=\operatorname{setNext}_{p}$ is preceded by transition $\left(s_{5}\right.$, done $\left._{p}, s_{5}^{\prime}\right)$ with $s_{5} \cdot \mathrm{NEXT}_{p}=$ $i_{2}$, we have a contradiction since $i_{2} \in t_{5}$. DONE and $t_{2}$. DONE $q$ is computed by gatherDone ${ }_{q}$ actions that are preceded by state $t_{5}$, which results in $i_{2} \in t_{2} . \mathrm{DONE}_{q}$. If there exists no such transition we have again a contradiction since we cannot have a collision in job $i_{2}$ at state $\tilde{s}_{2}$ as defined in Definition 5.2

The case $s_{1}<t_{4}$ is symmetric and can be proved with similar arguments.

From the discussion above we have that $t_{1}<s_{4}$, thus $t_{1}$. DONE $q \subseteq s_{4}$. DONE. Moreover $s_{3} \cdot \mathrm{DONE}_{p}$ is computed by gatherDone ${ }_{p}$ actions that are preceded by state $s_{4}$, from which we have that $t_{1} \cdot \mathrm{DONE}_{q} \subseteq s_{3} \cdot \mathrm{DONE}_{p}$. Since $s_{1}<s_{3}$ it holds that $s_{1} \cdot \mathrm{DONE}_{p} \subseteq s_{3}$. DONE ${ }_{p}$, thus we have that $s_{1} \cdot \mathrm{DONE}_{p} \cup t_{1} . \mathrm{DONE}_{q} \subseteq s_{3} . \mathrm{DONE}_{p}$. From $s_{1}<t_{4}$, with similar arguments as before, we can prove that $s_{1} \cdot \mathrm{DONE}_{p} \cup t_{1} \cdot \mathrm{DONE}_{q} \subseteq t_{3} \cdot \mathrm{DONE}_{q}$, which gives us that:

$$
s_{1} \cdot \mathrm{DONE}_{p} \cup t_{1} \cdot \mathrm{DONE}_{q} \subseteq s_{3} \cdot \mathrm{DONE}_{p} \cap t_{3} \cdot \mathrm{DONE}_{q}
$$

Now it only remains to prove that:

$$
\left|s_{3} \cdot \mathrm{DONE}_{p} \cup t_{3} \cdot \mathrm{DONE}_{q}\right|-\left|s_{1} \cdot \mathrm{DONE}_{p} \cup t_{1} \cdot \mathrm{DONE}_{q}\right|>m \cdot|q-p|
$$

If $p<q$ from Lemma 5.1 we have that $\left|s_{3} . \mathrm{DONE}_{p} \cap \overline{t_{3} . \mathrm{DONE}_{q}}\right|>(q-p) m$ or $\left|\overline{s_{3} \cdot \mathrm{DONE}_{p}} \cap t_{3} \cdot \mathrm{DONE}_{q}\right|>(q-p) m$. Since $s_{1} \cdot \mathrm{DONE}_{p} \cup t_{1} \cdot \mathrm{DONE}_{q} \subseteq s_{3} \cdot \mathrm{DONE}_{p} \cap$ $t_{3}$. $\mathrm{DONE}_{q}$, we have that:

$$
\left|s_{3} \cdot \mathrm{DONE}_{p} \cup t_{3} \cdot \mathrm{DONE}_{q}\right|-\left|s_{1} \cdot \mathrm{DONE}_{p} \cup t_{1} \cdot \mathrm{DONE}_{q}\right|>(q-p) \cdot m
$$

If $q<p$ with similar arguments we have that:

$$
\left|s_{3} \cdot \mathrm{DONE}_{p} \cup t_{3} \cdot \mathrm{DONE}_{q}\right|-\left|s_{1} \cdot \mathrm{DONE}_{p} \cup t_{1} \cdot \mathrm{DONE}_{q}\right|>(p-q) \cdot m
$$

Combining the above we have:

$$
\left|s_{3} \cdot \mathrm{DONE}_{p} \cup t_{3} \cdot \mathrm{DONE}_{q}\right|-\left|s_{1} \cdot \mathrm{DONE}_{p} \cup t_{1} \cdot \mathrm{DONE}_{q}\right|>m \cdot|q-p|
$$


Next we prove that a process $p$ cannot collide with a process $q$ more than $2\left\lceil\frac{n}{m \cdot|q-p|}\right\rceil$ times in any execution.

Lemma 5.5. If $\beta \geq 3 m^{2}$ there exists no execution $\alpha \in \operatorname{execs}\left(\mathrm{KK}_{\beta}\right)$ at which process $p$ collided with process $q$ in more than $2\left\lceil\frac{n}{m|q-p|}\right\rceil$ states according to Definition 5.2

Proof. Let execution $\alpha \in \operatorname{execs}\left(\mathrm{KK}_{\beta}\right)$ be an execution at which process $p$ collided with process $q$ in at least $2\left\lceil\frac{n}{m|q-p|}\right\rceil+1$ states. Let us examine the first $2\left\lceil\frac{n}{m|q-p|}\right\rceil+1$ such states. Let those states be $\tilde{s}_{1}<\tilde{s}_{2}<\ldots<\tilde{s}_{2\left\lceil\frac{n}{m|q-p|}\right\rceil}<\tilde{s}_{2\left\lceil\frac{n}{m|q-p|}\right\rceil+1}$. From Lemma 5.2 we have that there exists states $s_{1}<s_{2}<\ldots<s_{2\left\lceil\frac{n}{m \mid q-p}\right\rceil}<s_{2\left\lceil\left\lceil\frac{n}{m \mid q-p}\right\rceil+1\right.}$ that enable the compNext actions and states $t_{1}<t_{2}<\ldots<t_{2\left\lceil\frac{n}{m|q-p|}\right\rceil}<t_{2\left\lceil\frac{n}{m|q-p|}\right\rceil+1}$ that enable the compNext actions that lead to the collisions in states $\tilde{s}_{1}<\tilde{s}_{2}<\ldots<\tilde{s}_{2\left\lceil\frac{n}{m|q-p|}\right\rceil}<\tilde{s}_{2\left\lceil\frac{n}{m \mid q-p}\right\rceil+1}$. Then from Lemma 5.4 we have that $\forall i \in\left\{1, \ldots,\left\lceil\frac{n}{m|q-p|}\right\rceil\right\}$ :

$$
\begin{gathered}
\left|s_{2 i+1} . \mathrm{DONE}_{p} \cup t_{2 i+1} . \mathrm{DONE}_{q}\right|-\left|s_{2 i-1} . \mathrm{DONE}_{p} \cup t_{2 i-1} . \mathrm{DONE}_{q}\right|>m|q-p| \\
\left|s_{2 i+1} . \mathrm{DONE}_{p} \cup t_{2 i+1} . \mathrm{DONE}_{q}\right|-\left|s_{1} . \mathrm{DONE}_{p} \cup t_{1} . \mathrm{DONE}_{q}\right|>i m|q-p| \\
\left|s_{2 i+1} . \mathrm{DONE}_{p} \cup t_{2 i+1} . \mathrm{DONE}_{q}\right|>i m|q-p|
\end{gathered}
$$

From eq. 5 we have that:

$$
\left|s_{2\left\lceil\frac{n}{m|q-p|}\right\rceil+1} \cdot \mathrm{DONE}_{p} \cup t_{2\left\lceil\frac{n}{m|q-p|}\right\rceil+1} \cdot \mathrm{DONE}_{q}\right|>m|q-p|\left\lceil\frac{n}{m|q-p|}\right\rceil \geq n
$$

Equation 6 leads to a contradiction since $s_{2\left\lceil\frac{n}{m|q-p|}\right\rceil+1} . \mathrm{DONE}_{p} \cup t_{2\left\lceil\frac{n}{m|q-p|}\right\rceil+1} . \mathrm{DONE}_{q} \subseteq \mathcal{J}$ and $|\mathcal{J}|=n$.

Finally we are ready to prove the main theorem on the work complexity of algorithm $\mathrm{KK}_{\beta}$ for $\beta \geq 3 m^{2}$.

Theorem 5.6. If $\beta \geq 3 m^{2}$ algorithm $\mathrm{KK}_{\beta}$ has work complexity $W_{\mathrm{KK}_{\beta}}=\mathrm{O}(n m \log n \log m)$.

Proof. We start with the observation that in any execution $\alpha$ of algorithm $\mathrm{KK}_{\beta}$, if there exists process $p$, job $i$, transition $\left(s_{1}\right.$, done $\left._{p}, s_{1}^{\prime}\right)$ and $j \in\{1, \ldots, n\}$ such that $s_{1} \cdot \operatorname{POS}_{p}(p)=j$, $s_{1} \cdot \mathrm{NEXT}_{p}=i$, for any process $q \neq p$ there exists at most one transition $\left(t_{1}\right.$, gatherDone $\left._{q}, t_{1}^{\prime}\right)$ in $\alpha$, with $t_{1} \cdot \mathrm{Q}_{q}=p, t_{1} \cdot \operatorname{POS}_{q}(p)=j$ and $t_{1} \geq s_{1}$. Such transition performs exactly one read operation from the shared memory, one insertion at the set $\mathrm{DONE}_{q}$ and one removal from the set $\mathrm{FREE}_{q}$, thus such a transition costs $\mathrm{O}(\log n)$ work. Clearly there exist at most $m-1$ such transitions for each done $e_{p}$. From Lemma 4.1 for all processes there can be at most $n$ actions done $_{p}$ in any execution $\alpha$ of algorithm $\mathrm{KK}_{\beta}$. Each done $\mathrm{p}_{p}$ action performs one write operation in shared memory, one insertion at the set $\mathrm{DONE}_{p}$ and one removal from the set $\mathrm{FREE}_{p}$, thus

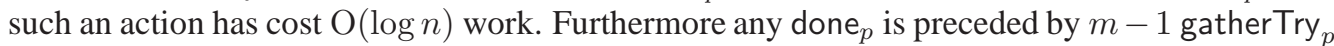
read actions that read the next array and each add at most one element to the set $\mathrm{TRY}_{p}$ with 
cost $\mathrm{O}(\log n)$ and $m-1$ gatherDone ${ }_{p}$ read actions that do not add elements in the $\mathrm{DONE}_{p}$ set. Note that we have already counted the gatherDone ${ }_{p}$ read actions that result in adding jobs at the $\mathrm{DONE}_{p}$ set. Finally any done $e_{p}$ action is preceded by one compNext ${ }_{p}$ action. This action is dominated by the cost of the $\operatorname{rank}\left(\mathrm{FREE}_{p}, \mathrm{TRY}_{p}, i\right)$ function. If the sets $\mathrm{FREE}_{p}, \mathrm{TRY}_{p}$ are represented with some efficient tree structure like red-black tree or some variant of $B$-tree [5, 19] that allows insertion, deletion and search of an element in $\mathrm{O}(\log n)$, an invocation of function $\operatorname{rank}\left(\mathrm{FREE}_{p}, \mathrm{TRY}_{p}, i\right) \operatorname{costs} \mathrm{O}(m \log n)$ work. That gives us a total of $\mathrm{O}(n m \log n)$ work associated with the done $e_{p}$ actions.

If a process $p$ collided with a process $q$ in job $i$ at state $s$, we have an extra compNext ${ }_{p}$ action, $m-1$ extra gatherTry ${ }_{p}$ read actions and insertions in the $\mathrm{TRY}_{p}$ set and $m-1$ gatherDone ${ }_{p}$ read actions that do not add elements in the $\mathrm{DONE}_{p}$ set. Thus each collision costs $\mathrm{O}(m \log n)$ work. Since $\beta \geq 3 \mathrm{~m}^{2}$ from Lemma 5.5 for two distinct processes $p, q$ we have that in any execution $\alpha$ of algorithm $\mathrm{KK}_{\beta}$ there exist less than $2\left\lceil\frac{n}{m|q-p|}\right\rceil$ collisions. For process $p$ if we count all such collisions with any other process $q$ we get:

$$
\begin{gathered}
\sum_{q \in \mathcal{P}-\{p\}} 2\left\lceil\frac{n}{m|q-p|}\right] \leq 2(m-1)+\frac{2 n}{m} \sum_{q \in \mathcal{P}-\{p\}} \frac{1}{|q-p|} \leq \\
\leq 2(m-1)+\frac{4 n}{m} \sum_{i=1}^{\left\lceil\frac{m}{2}\right\rceil} \frac{1}{i} \leq 2(m-1)+\frac{4 n}{m} \log m
\end{gathered}
$$

If we count the total number of collisions for all the $m$ processes we get that if $\beta \geq 3 m^{2}$ in any execution of algorithm $\mathrm{KK}_{\beta}$ there can be at most $2 m^{2}+4 n \log m<4(n+1) \log m$ collisions (since $n>\beta)$. Thus collisions $\operatorname{cost} \mathrm{O}(n m \log n \log m)$ work. Finally any process $p$ that fails may add in the work complexity less than $\mathrm{O}(m \log n)$ work from its compNext ${ }_{p}$ action and from reads (if the process fails without performing a done ${ }_{p}$ action after its latest compNext ${ }_{p}$ action). So for the work complexity of algorithm $\mathrm{KK}_{\beta}$ if $\beta \geq 3 m^{2}$ we have that $W_{\mathrm{KK}_{\beta}}=\mathrm{O}(n m \log n \log m)$.

\section{An Asymptotically Work Optimal Algorithm}

We demonstrate how to solve the at-most-once problem with effectiveness $n$ $\mathrm{O}\left(m^{2} \log n \log m\right)$ and work complexity $\mathrm{O}\left(n+m^{(3+\epsilon)} \log n\right)$, for any constant $\epsilon>0$, such that $1 / \epsilon$ is a positive integer, when $m=\mathrm{O}(\sqrt[3]{n})$, using algorithm $\mathrm{KK}_{\beta}$ with $\beta=3 m^{2}$. Algorithm IterativeKK $(\epsilon)$, presented in Fig.3, performs iterative calls to a variation of algorithm $\mathrm{KK}_{\beta}$, called IterStepKK. IterativeKK $(\epsilon)$ has $3+1 / \epsilon$ distinct matrices done and vectors next in shared memory, with different granularities. One done matrix, stores the regular jobs performed, while the remaining $2+1 / \epsilon$ matrices store super-jobs. Super-jobs are groups of consecutive jobs. From them, one stores super-jobs of size $m \log n \log m$, while the remaining $1+1 / \epsilon$ matrices, store super-jobs of size $m^{1-i \epsilon} \log n \log ^{1+i} m$ for $i \in\{1, \ldots, 1 / \epsilon\}$. The $3+1 / \epsilon$ distinct vectors next are used in a similar way as the matrices done.

The algorithm IterStepKK is different from $\mathrm{KK}_{\beta}$ in the following ways. First, all instances of IterStepKK work for $\beta=3 \mathrm{~m}^{2}$. Moreover, IterStepKK has a termination flag in shared memory. This termination flag is initially 0 and is set to 1 by any process that decides to terminate. In the execution of algorithm IterStepKK, a process $p$, that in an action compNext has $\left|\mathrm{FREE}_{p} \backslash \mathrm{TRY}_{p}\right|<3 m^{2}$, sets the termination flag to 1 , computes new sets $\mathrm{FREE}_{p}$ and 


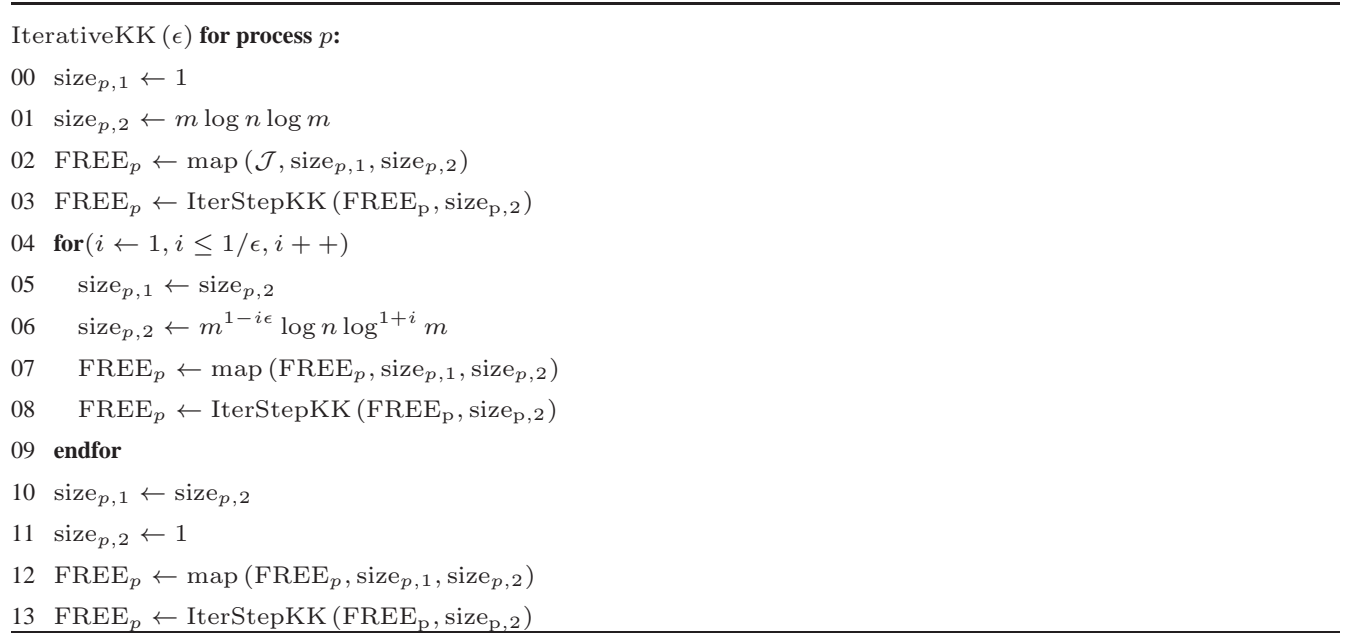

Figure 3: Algorithm IterativeKK $(\epsilon)$ : pseudocode

$\mathrm{TRY}_{p}$, returns the set $\mathrm{FREE}_{p} \backslash \mathrm{TRY}_{p}$ and terminates. After a process $p$ checks if it is safe to perform a job, the process also checks the termination flag and if the flag is 1 , the process instead of performing the job, computes new sets $\mathrm{FREE}_{p}$ and $\mathrm{TRY}_{p}$, returns the set $\mathrm{FREE}_{p} \backslash \mathrm{TRY}_{p}$ and terminates. Finally, algorithm IterStepKK takes as inputs the variable size and a set $\mathrm{SET}_{1}$, such that $\left|\mathrm{SET}_{1}\right|>3 m^{2}$, and returns the set $\mathrm{SET}_{2}$ as output. $\mathrm{SET}_{1}$ contains super-jobs of size size. In IterStepKK, with an action $\mathrm{do}_{p, j}$ process $p$ performs all the jobs of super-job $j$. A process $p$ performs as many super-jobs as it can and returns in $\mathrm{SET}_{2}$ the super-jobs it can verify that no process will perform.

In algorithm IterativeKK $(\epsilon)$ we use also the function $\mathrm{SET}_{2}=\operatorname{map}\left(\mathrm{SET}_{1}, \mathrm{size}_{1}, \mathrm{size}_{2}\right)$, that takes the set of super-jobs $\mathrm{SET}_{1}$, with super-jobs of size size $e_{1}$ and maps it to a set of superjobs $\mathrm{SET}_{2}$ with size $s_{i z e_{2}}$. A job $i$ is always mapped to the same super-job of a specific size and there is no intersection between the jobs in super-jobs of the same size.

\subsection{Analysis}

We begin the analysis of algorithm IterativeKK $(\epsilon)$ by showing in Theorem 6.3 that IterativeKK $(\epsilon)$ solves the at-most-once problem. This is done by first showing in Lemma 6.1 that algorithm IterStepKK solves the at-most-once problem for the set of all super-jobs of a specific size, and then by showing in Lemma 6.2 that there exist no performed super-jobs in any output set $\mathrm{SET}_{2}$. We complete the analysis with Theorem 6.4 where we show that algorithm IterativeKK $(\epsilon)$ has effectiveness $E_{\text {IterativeKK }(\epsilon)}(n, m, f)=n-\mathrm{O}\left(m^{2} \log n \log m\right)$ and work complexity $W_{\text {IterativeKK }(\epsilon)}=\mathrm{O}\left(n+m^{3+\epsilon} \log n\right)$.

Let the set of all super-jobs of a specific size $d$ be SuperSet $_{d}$. All invocations of algorithm IterStepKK on sets $\mathrm{SET}_{1} \subseteq$ SuperSet $_{d}$, use the matrix done and vector next that correspond to the super-jobs of size $d$. Moreover each process $p$ invokes algorithm IterStepKK for a set $\mathrm{SET}_{1} \subseteq$ SuperSet $_{d}$ only once. We have the following lemma.

Lemma 6.1. Algorithm IterStepKK solves the at-most-once problem for the set SuperSet $_{d}$.

Proof. As described above, algorithm IterStepKK is different from $\mathrm{KK}_{\beta}$ in the following ways: 
- Process $p$, on algorithm IterStepKK, has an input set $\mathrm{SET}_{1} \subseteq$ SuperSet $_{d}$ of super-jobs of size $d$ to be performed and outputs a set $\mathrm{SET}_{2} \subset$ SuperSet $_{d}$ of super-jobs, that have not been performed. Process $p$ initially sets its set $\mathrm{FREE}_{p}$, equal to $\mathrm{SET}_{1}$ and proceeds as it would do when executing $\mathrm{KK}_{\beta}$, with the difference that an action do $\mathrm{o}_{p, i}$ results in performing all the jobs under super-job $i$. Entries in the matrix done and vector next in shared memory correspond to the identifiers of super-jobs of set SuperSet ${ }_{d}$. Again after its initialization, entries are only removed from set $\mathrm{FREE}_{p}$.

Note that the main difference caused by this modification, between algorithm IterStepKK and algorithm $\mathrm{KK}_{\beta}$, is that jobs are replaced by super-jobs, and that the initial sets $\mathrm{FREE}_{p}$ and $\mathrm{FREE}_{q}$ of processes $p, q$ could be set to different subsets of set SuperSet . This does not affect the correctness of the algorithm, since in any state $s$ of an execution $\alpha$ of algorithm $\mathrm{KK}_{\beta}$, the sets $\mathrm{FREE}_{p}$ and $\mathrm{FREE}_{q}$ could be different subsets of the set of all jobs $\mathcal{J}$.

- Algorithm IterStepKK has a termination flag in shared memory. The termination flag is initially 0 and is set to 1 by any process that decides to terminate. As mentioned above, any process that discovers that $\left|\mathrm{FREE}_{p} \backslash \mathrm{TRY}_{p}\right|<3 m^{2}$ in an action compNext ${ }_{p}$, sets the termination flag to 1 , computes new sets $\mathrm{FREE}_{p}$ and $\mathrm{TRY}_{p}$, returns the set $\mathrm{FREE}_{p} \backslash$ $\mathrm{TRY}_{p}$ and terminates. This modification only affects the sequence of actions during the termination of a process $p$. Observe process $p$ does not perform any super-jobs in that termination sequence.

Additionally, after a process $p$ checks if it is safe to perform a super-job, it also checks the termination flag and if the flag is 1 , the process instead of performing the super-job, enters the termination sequence, computing new sets $\mathrm{FREE}_{p}$ and $\mathrm{TRY}_{p}$, returning the set $\mathrm{FREE}_{p} \backslash \mathrm{TRY}_{p}$ and terminating. A process $p$ first checks if it is safe to perform a super-job according to algorithm $\mathrm{KK}_{\beta}$ and then checks the flag. Thus this modification only affects the effectiveness, but not the correctness of the algorithm, since it could only result in a super-job that was safe to perform not being performed.

- Finally all instances of IterStepKK work for $\beta=3 m^{2}$. This does not affect correctness, since Lemma4.1 holds for any $\beta$.

It is easy to see that none of the modifications described above affect the key arguments in the proof of Lemma4.1 Thus with similar arguments as in the proof of Lemma4.1, we can show that there exists no execution of algorithm IterStepKK, where two distinct actions $\pi=\mathrm{do}_{p, i}$ and $\pi^{\prime}=$ do $_{q, i}$ take place for a super-job $i \in$ SuperSet $_{d}$ and processes $p, q \in \mathcal{P}$ ( $p$ could be equal to $q$ ).

Next we show that in the output sets of algorithm IterStepKK at a specific iteration (calls for super-jobs of size $d$ ), no completed super-jobs are included. Combined with the previous lemma, this argument will help us establish that algorithm IterativeKK $(\epsilon)$ solves that at-mostonce problem.

Lemma 6.2. There exists no execution $\alpha$ of algorithm IterStepKK, such that there exists action $\mathrm{do}_{q, i} \in \alpha$ for some process $q$ and super-job $i$ in the output set $\mathrm{SET}_{2} \subset$ SuperSet $_{d}$ of some process $p$ ( $p$ could be equal to process $q$ ).

Proof. As described above, a process $p$ before terminating algorithm IterStepKK, either sets the flag to 1 or observes that the flag is set to 1 . The process $p$ then computes new sets $\mathrm{FREE}_{p}$ and 
$\mathrm{TRY}_{p}$, returns the set $\mathrm{FREE}_{p} \backslash \mathrm{TRY}_{p}$ and terminates its execution of algorithm IterStepKK for input set $\mathrm{SET}_{1} \subseteq$ SuperSet $_{d}$ and super-jobs of size $d$. Let state $s$ be the state at which process $p$ terminates, we have that $\mathrm{SET}_{2}=s \cdot \mathrm{FREE}_{p} \backslash s \cdot \mathrm{TRY}_{p}$. If $p=q$ and there exists action $\pi=\mathrm{do}_{p, i}$ in execution $\alpha$ of algorithm IterStepKK, for super-jobs $i \in$ SuperSet $_{d}$, clearly $\pi<s$, from which we have that $i \notin s . \mathrm{FREE}_{p}$ and thus $i \notin \mathrm{SET}_{2}$.

It is easy to see that if $p \neq q$ and $i \in \mathrm{SET}_{2}$ of process $p$, there exists no action $\pi=\mathrm{do}_{q, i}$ in execution $\alpha$. If $i \in \mathrm{SET}_{2}$ then $i \in s \cdot \mathrm{FREE}_{p}$ and $i \notin s . \mathrm{TRY}_{p}$. Moreover process $p$ either set flag to 1 or observed that the flag was set, before computing sets $s \cdot \mathrm{FREE}_{p}$ and $s . \mathrm{TRY}_{p}$. If there exists $\pi=\mathrm{do}_{q, i} \in \alpha$, for process $q$, it must be the case that after process $q$ performed the transition $\left(t\right.$, compNext $\left._{q}, t^{\prime}\right) \mapsto \pi$ (see Definition5.1 of immediate predecessor), it read the flag and found it was equal to 0 . This leads to a contradiction, since it must be the case that either $i \in s . \mathrm{TRY}_{p}$ or $i \notin s . \mathrm{FREE}_{p}$.

We are ready now to show the correctness of algorithm IterativeKK $(\epsilon)$.

Theorem 6.3. Algorithm IterativeKK $(\epsilon)$ solves the at-most-once problem.

Proof. From Lemma 6.1 we have that any super-job of a specific size $d$ is performed at-mostonce (if performed at all) in the execution of algorithm IterStepKK for the super-jobs in the

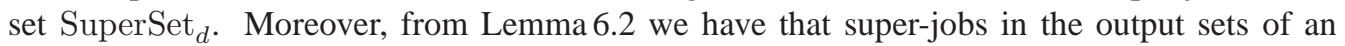
execution of algorithm IterStepKK for super-jobs of size $d$, have not been performed. Function $\mathrm{SET}_{2}=\operatorname{map}\left(\mathrm{SET}_{1}\right.$, size $_{1}$, size $\left._{2}\right)$ maps the jobs in the super-jobs of set $\mathrm{SET}_{1}$, to super-jobs in $\mathrm{SET}_{2}$. A job $i$ is always mapped to the same super-job of a specific size $d$ and there is no

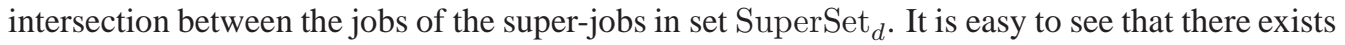
no execution of algorithm IterativeKK $(\epsilon)$, where a job $i$ is performed more than once.

We complete the analysis of algorithm IterativeKK $(\epsilon)$ with Theorem 6.4, which gives upper bounds for the effectiveness and work complexity of the algorithm.

Theorem 6.4. Algorithm IterativeKK $(\epsilon)$ has $W_{\text {IterativeKK }(\epsilon)}=\mathrm{O}\left(n+m^{3+\epsilon} \log n\right)$ work complexity and effectiveness $E_{\text {IterativeKK }(\epsilon)}(n, m, f)=n-\mathrm{O}\left(m^{2} \log n \log m\right)$.

Proof. In order to determine the effectiveness and work complexity of algorithm IterativeKK $(\epsilon)$, we compute the jobs performed by and the work spent in each invocation of IterStepKK. Moreover we compute the work that the invocations to the function map () add.

The first invocation to function map () in line 02 can be completed by process $p$ with work $\mathrm{O}\left(\frac{n}{m \log n \log m} \log n\right)$, since process $p$ needs to construct a tree with $\frac{n}{m \log n \log m}$ elements. This contributes for all processes $\mathrm{O}\left(\frac{n}{\log m}\right)$ work. From Theorem 5.6 we have that IterStepKK in 03 has total work $\mathrm{O}\left(n+\frac{n}{m \log n \log m} m \log n \log m\right)=\mathrm{O}(n)$, where the first $n$ comes from do actions and the second term from the work complexity of Theorem 5.6. Note that we count $\mathrm{O}(1)$ work for each normal job executed by a do action on a super-job. That means that in the invocation of IterStepKK in line 03 , do actions cost $m \log n \log m$ work. Moreover from Theorem 4.4 we have effectiveness $\frac{n}{m \log n \log m}-\left(3 m^{2}+m-2\right)$ on the super-jobs of size $m \log n \log m$. From the super-jobs not completed, up to $m-1$ may be contained in the $\mathrm{TRY}_{p}$ sets upon termination in line 03. Since those super-jobs are not added (and thus are ignored) in the output $\mathrm{FREE}_{p}$ set in line 03, up to $(m-1) m \log n \log m$ jobs may not be performed by IterativeKK $(\epsilon)$. The set $\mathrm{FREE}_{p}$ returned by algorithm IterStepKK in line 03 has no more than $3 m^{2}+m-2$ super-jobs of size $m \log n \log m$. 
In each repetition of the loop in lines $04-09$, the map () function in line 07 constructs a FREE $_{p}$ set with at most $\mathrm{O}\left(m^{2+\epsilon} / \log m\right)$ elements, which costs $\mathrm{O}\left(m^{2+\epsilon}\right)$ per process $p$ for a total of $\mathrm{O}\left(\mathrm{m}^{3+\epsilon}\right)$ work for all processes. Moreover each invocation of IterStepKK in line 08 costs $\mathrm{O}\left(3 m^{3} \log n \log m+m^{3+\epsilon} \log m\right)<\mathrm{O}\left(m^{3+\epsilon} \log n\right)$ work from Theorem 5.6 where the term $3 m^{3} \log n \log m$ is an upper bound on the work needed for the do actions on the super-jobs. From Theorem 4.4 we have that each output $\mathrm{FREE}_{p}$ set in line 08 has at most $3 m^{2}+m-2$ super-jobs. Moreover from each invocation of IterStepKK in line 08 at most $m-1$ super-jobs are lost in TRY sets. Those account for less than $(m-1) m \log n \log m$ jobs in each iteration, since the size of the super-jobs in the iterations of the loop in lines $04-09$ is strictly less than $m \log n \log m$.

When we leave the loop in lines $04-09$, we have a $\mathrm{FREE}_{p}$ set with at most $3 m^{2}+m-2$ super-jobs of size $\log n \log ^{1+1 / \epsilon} m$, which means that in line 12 function map () will return a set $\mathrm{FREE}_{p}$ with less than $\left(3 m^{2}+m-2\right)\left(\log n \log ^{1+1 / \epsilon} m\right)$ elements that correspond to jobs and not super-jobs. This costs for all processes a total of $\mathrm{O}\left(m^{3} \log m \log \log n \log \log m\right)<$ $\mathrm{O}\left(m^{3+\epsilon} \log n\right)$ work, since $\epsilon$ is a constant. Finally we have that IterStepKK in line 13 has from Theorem 5.6 work $\mathrm{O}\left(m^{3} \log ^{2} m \log \log n \log \log m\right)<\mathrm{O}\left(m^{3+\epsilon} \log n\right)$ and from Theorem 4.4 effectiveness $\left(3 m^{2}+m-2\right)\left(\log n \log ^{1+1 / \epsilon} m\right)-\left(3 m^{2}+m-2\right)$.

If we add up all the work, we have that $W_{\text {IterativeKK }(\epsilon)}=\mathrm{O}\left(n+m^{3+\epsilon} \log n\right)$ since the loop in lines $04-09$ repeats $1+1 / \epsilon$ times and $\epsilon$ is a constant. Moreover for the effectiveness, we have that less than or equal to $(m-1) m \log n \log m$ jobs will be lost in the TRY set at line 03 . After that strictly less than $(m-1) m \log n \log m$ jobs will be lost in the TRY sets of the iterations of the loop in lines $04-09$ and fewer than $3 m^{2}+m-2$ jobs will be lost from the effectiveness of the last invocation of IterStepKK in line 13. Thus we have that $E_{\operatorname{IterativeKK}(\epsilon)}(n, m, f)=$ $n-\mathrm{O}\left(m^{2} \log n \log m\right)$.

For any $m=\mathrm{O}(\sqrt[3+\epsilon]{n / \log n})$, algorithm IterativeKK $(\epsilon)$ is work optimal and asymptotically effectiveness optimal.

\section{An Asymptotically Optimal Algorithm for the Write-All Problem}

Based on IterativeKK $(\epsilon)$ we construct algorithm WA_IterativeKK $(\epsilon)$ Fig.4 that solves the Write-All problem [23] with work complexity $\mathrm{O}\left(n+m^{(3+\epsilon)} \log n\right)$, for any constant $\epsilon>0$, such that $1 / \epsilon$ is a positive integer. From Kanellakis and Shvartsman [23] the Write-All problem for the shared memory model, consists of: "Using $m$ processors write 1's to all locations of an array of size $n . "$ The problem assumes that all cells of the array are initialized to 0. Algorithm WA_IterativeKK $(\epsilon)$ is different from IterativeKK $(\epsilon)$ in two ways. It uses a modified version of IterStepKK, that instead of returning the $\mathrm{FREE}_{p} \backslash \mathrm{TRY}_{p}$ set upon termination returns the set $\mathrm{FREE}_{p}$ instead. Let us name this modified version WA_IterStepKK. Moreover in WA_IterativeKK $(\epsilon)$ after line 13, process $p$, instead of terminating, executes all jobs in the set $\mathrm{FREE}_{p}$. Note that since we are interested in the Write-All problem, when process $p$ performs a job $i$ with action $\mathrm{do}_{p, i}$, process $p$ just writes 1 , in the $i$-th position of the Write All array $w a[1, \ldots, n]$ in shared memory.

Theorem 7.1. Algorithm WA_IterativeKK $(\epsilon)$ solves the Write-All problem with work complexity $W_{\text {WA_IterativeKK }(\epsilon)}=\mathrm{O}\left(n+m^{3+\epsilon} \log n\right)$.

Proof. We prove this with similar arguments as in the proof of Theorem 6.4 From Theorem 4.4 after each invocation of WA_IterStepKK the output set $\mathrm{FREE}_{p}$ has less than $3 m^{2}+m-1$ 


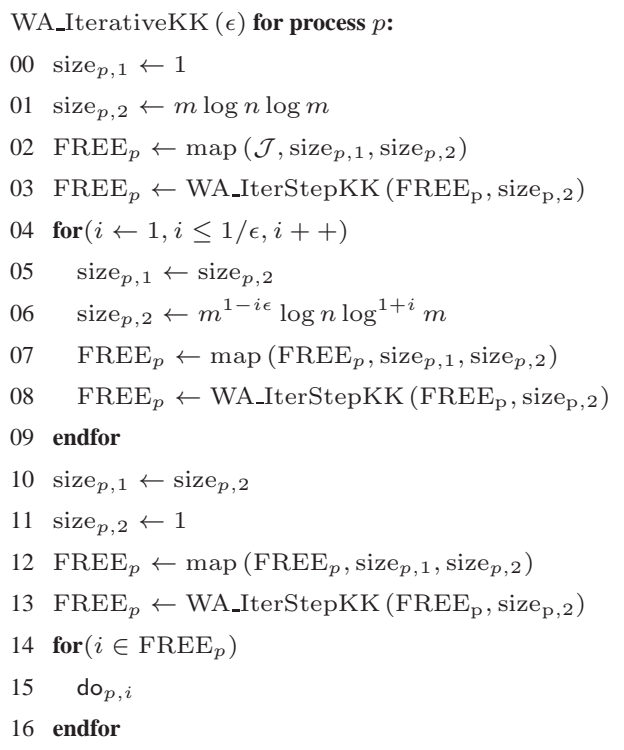

Figure 4: Algorithm WA_IterativeKK $(\epsilon)$ : pseudocode

super-jobs. The difference is that now we do not leave jobs in the $\mathrm{TRY}_{p}$ sets, since we are not interested in maintaining the at-most-once property between successive invocations of algorithm WA_IterStepKK. Since after each invocation of WA_IterStepKK the output set $\mathrm{FREE}_{p}$ has the same upper bound on super-jobs as in IterativeKK $(\epsilon)$, with similar arguments as in the proof of Theorem 6.4 we have that at line 13 the total work performed by all processes is $\mathrm{O}\left(n+m^{3+\epsilon} \log n\right)$. Moreover from Theorem 4.4 the output $\mathrm{FREE}_{p}$ set in line $p$ has less than $3 m^{2}+m-2$ jobs. This gives us for all processes a total work of $\mathrm{O}\left(\mathrm{m}^{3}\right)$ for the loop in lines $14-16$. After the loop in lines $14-16$ all jobs have been performed, since we left no TRY sets behind, thus algorithm WA_IterativeKK $(\epsilon)$ solves the Write-All problem with work complexity $W_{\text {WA_IterativeKK }(\epsilon)}=\mathrm{O}\left(n+m^{3+\epsilon} \log n\right)$.

For any $m=\mathrm{O}(\sqrt[3+\epsilon]{n / \log n})$, algorithm WA_IterativeKK $(\epsilon)$ is work optimal.

\section{Conclusions}

We devised and analyzed a deterministic algorithm for the at most once problem called $\mathrm{KK}_{\beta}$. For $\beta=m$ algorithm $\mathrm{KK}_{\beta}$ has effectiveness $n-2 m+2$, which is asymptotically optimal for any $m=\mathrm{o}(n)$ and close by an additive factor of $m$ to the effectiveness upper bound $n-m+1$ on all possible algorithms. This is a significant improvement over the previous best known deterministic algorithm [26], that achieves asymptotically optimal effectiveness only for $m=\mathrm{O}(1)$. With respect to work complexity, for any constant $\epsilon$ and for $m=\mathrm{O}(\sqrt[3+\epsilon]{n / \log n})$ we demonstrate how to use $\mathrm{KK}_{\beta}$ with $\beta=3 \mathrm{~m}^{2}$, in order to construct an iterated algorithm IterativeKK $(\epsilon)$, that is work-optimal and asymptotically effectiveness-optimal. Finally we used algorithm IterativeKK $(\epsilon)$ in order to solve the Write-All problem with work complexity $\mathrm{O}\left(n+m^{(3+\epsilon)} \log n\right)$, for any constant $\epsilon>0$, which is work optimal for $m=\mathrm{O}(\sqrt[3+\epsilon]{n / \log n})$. Our solution improves on the algorithm of Malewicz [36] both in terms of the range of pro- 
cessors for which we achieve optimal work and on the fact that we do not assume test-andset primitives, but use only atomic read/write shared memory. The solution of Kowalski and Shvartsman [28] is work optimal for a wider range of processors $m$ than our algorithm, but their algorithm uses a collection of $q$ permutations with contention $\mathrm{O}(q \log q)$. Although an efficient polynomial time construction of permutations with contention $\mathrm{O}(q$ polylog $q)$ has been developed by Kowalski et al. [27], constructing permutations with contention $\mathrm{O}(q \log q)$ in polynomial time is still an open problem. Subsequent to the conference version of this paper [25], Alistarh et al. [2] show that there exists a deterministic algorithm for the Write-All problem with work $\mathrm{O}\left(n+m \log ^{5} n \log ^{2} \max (n, m)\right)$, by derandomizing their randomized solution for the problem. Their solution is so far existential, while ours explicit.

In terms of open questions there still exists an effectiveness gap between the shown effectiveness of $n-2 m+2$ of algorithm $\mathrm{KK}_{\beta}$ and the known effectiveness bound of $n-m+1$. It would be interesting to see if this can be bridged for deterministic algorithms. Moreover, there is a lack of an upper bound on work complexity, when the effectiveness of an algorithm approaches the optimal. Finally it would be interesting to study the existence and efficiency of algorithms that try to implement at-most-once semantics in systems with different means of communication, such as message-passing systems.

\section{References}

[1] Y. Afek, E. Weisberger, and H. Weisman. A completeness theorem for a class of synchronization objects. In Proc. of the 12th annual ACM Symp. on Principles of Distributed Computing(PODC '93), pages 159-170. ACM, 1993.

[2] D. Alistarh, M. Bender, S. Gilbert, and R. Guerraoui. How to allocate tasks asynchronously. In Foundations of Computer Science (FOCS), 2012 IEEE 53rd Annual Symposium on, pages 331 -340, Oct. 2012.

[3] R. J. Anderson and H. Woll. Algorithms for the certified write-all problem. SIAM J. Computing, 26(5):1277-1283, 1997.

[4] H. Attiya, A. Bar-Noy, D. Dolev, D. Peleg, and R. Reischuk. Renaming in an asynchronous environment. J. ACM, 37(3):524-548, 1990.

[5] R. Bayer. Symmetric binary b-trees: Data structure and maintenance algorithms. Acta Informatica, 1:290-306, 1972.

[6] A. D. Birrell and B. J. Nelson. Implementing remote procedure calls. ACM Trans. Comput. Syst., 2(1):39-59, 1984.

[7] D. Bokal, B. Brešar, and J. Jerebic. A generalization of hungarian method and hall's theorem with applications in wireless sensor networks. Discrete Appl. Math., 160(4-5):460-470, Mar. 2012.

[8] S. Chaudhuri, B. A. Coan, and J. L. Welch. Using adaptive timeouts to achieve at-most-once message delivery. Distrib. Comput., 9(3):109-117, 1995.

[9] B. S. Chlebus and D. R. Kowalski. Cooperative asynchronous update of shared memory. In STOC, pages 733-739, 2005.

[10] A. Czygrinow, M. Hanćkowiak, E. Szymańska, and W. Wawrzyniak. Distributed 2-approximation algorithm for the semi-matching problem. In Proceedings of the 26th international conference on Distributed Computing, DISC'12, pages 210-222, Berlin, Heidelberg, 2012. Springer-Verlag.

[11] G. Di Crescenzo and A. Kiayias. Asynchronous perfectly secure communication over one-time pads. In Proc. of 32nd International Colloquium on Automata, Languages and Programming(ICALP '05), pages 216-227. Springer, 2005.

[12] A. Drucker, F. Kuhn, and R. Oshman. The communication complexity of distributed task allocation. In Proc. of the 31st annual Symp. on Principles of Distributed Computing(PODC '12), pages 67-76. ACM, 2012.

[13] M. J. Fischer, N. A. Lynch, and M. S. Paterson. Impossibility of distributed consensus with one faulty process. $J$. ACM, 32(2):374-382, 1985.

[14] M. Fitzi, J. B. Nielsen, and S. Wolf. How to share a key. In Allerton Conference on Communication, Control, and Computing 2007, 2007.

[15] C. Georgiou and A. A. Shvartsman. Do-All Computing in Distributed Systems: Cooperation in the Presence of Adversity. Springer, 2008.

[16] C. Georgiou and A. A. Shvartsman. Cooperative Task-Oriented Computing: Algorithms and Complexity. Synthesis Lectures on Distributed Computing Theory. Morgan \& Claypool Publishers, 2011. 
[17] K. J. Goldman and N. A. Lynch. Modelling shared state in a shared action model. In Logic in Computer Science, pages $450-463,1990$.

[18] J. Groote, W. Hesselink, S. Mauw, and R. Vermeulen. An algorithm for the asynchronous write-all problem based on process collision. Distributed Computing, 14(2):75-81, 2001.

[19] L. J. Guibas and R. Sedgewick. A dichromatic framework for balanced trees. In 19th Annual Symposium on Foundations of Computer Science(FOCS), pages 8-21, 1978.

[20] N. J. A. Harvey, R. E. Ladner, L. Lovász, and T. Tamir. Semi-matchings for bipartite graphs and load balancing. J. Algorithms, 59(1):53-78, Apr. 2006.

[21] M. Herlihy. Wait-free synchronization. ACM Transactions on Programming Languages and Systems, 13:124-149, 1991.

[22] K. C. Hillel. Multi-sided shared coins and randomized set-agreement. In Proc. of the 22nd ACM Symp. on Parallel Algorithms and Architectures (SPAA'10), pages 60-68, 2010.

[23] P. C. Kanellakis and A. A. Shvartsman. Fault-Tolerant Parallel Computaion. Kluwer Academic Publishers, 1997.

[24] S. Kentros, C. Kari, and A. Kiayias. The strong at-most-once problem. In Proc. of 26th International Symp. on Distributed Computing(DISC'12), pages 390-404, 2012.

[25] S. Kentros and A. Kiayias. Solving the at-most-once problem with nearly optimal effectiveness. In ICDCN, pages 122-137, 2012.

[26] S. Kentros, A. Kiayias, N. C. Nicolaou, and A. A. Shvartsman. At-most-once semantics in asynchronous shared memory. In Proc. of 23rd International Symp. on Distributed Computing(DISC'09), pages 258-273, 2009.

[27] D. Kowalski, P. M. Musial, and A. A. Shvartsman. Explicit combinatorial structures for cooperative distributed algorithms. In Proceedings of the 25th IEEE International Conference on Distributed Computing Systems, ICDCS '05, pages 49-58, Washington, DC, USA, 2005. IEEE Computer Society.

[28] D. R. Kowalski and A. A. Shvartsman. Writing-all deterministically and optimally using a nontrivial number of asynchronous processors. ACM Transactions on Algorithms, 4(3), 2008.

[29] L. Lamport. The part-time parliament. ACM Trans. Comput. Syst., 16(2):133-169, 1998.

[30] B. W. Lampson, N. A. Lynch, and J. F. S-Andersen. Correctness of at-most-once message delivery protocols. In Proc. of the IFIP TC6/WG6.1 6th International Conference on Formal Description Techniques(FORTE '93), pages 385-400. North-Holland Publishing Co., 1994.

[31] K.-J. Lin and J. D. Gannon. Atomic remote procedure call. IEEE Trans. Softw. Eng., 11(10):1126-1135, 1985.

[32] B. Liskov. Distributed programming in argus. Commun. ACM, 31(3):300-312, 1988.

[33] B. Liskov, L. Shrira, and J. Wroclawski. Efficient at-most-once messages based on synchronized clocks. ACM Trans. Comput. Syst., 9(2):125-142, 1991.

[34] N. Lynch and M. Tuttle. An introduction to input/output automata. CWI-Quarterly, pages 219-246, 1989.

[35] N. A. Lynch. Distributed Algorithms. Morgan Kaufmann Publishers, 1996.

[36] G. Malewicz. A work-optimal deterministic algorithm for the certified write-all problem with a nontrivial number of asynchronous processors. SIAM J. Comput., 34(4):993-1024, 2005.

[37] A. Z. Spector. Performing remote operations efficiently on a local computer network. Commun. ACM, 25(4):246260, 1982.

[38] R. W. Watson. The delta-t transport protocol: Features and experience. In Proc. of the 14th Conf. on Local Computer Networks, pages 399-407, 1989. 